\title{
Uphill energy transfer mechanism for photosynthesis in the Antarctic alga
}

\section{Makiko Kosugi ( $\nabla$ kosugi@bio.chuo-u.ac.jp)}

National Institutes of Natural Science https://orcid.org/0000-0002-7415-1536

\section{Masato Kawasaki}

High Energy Accelerator Research Organization (KEK)

\section{Yutaka Shibata}

Tohoku University

\section{Kojiro Hara}

Akita Prefectural University

\section{Shinichi Takaichi}

Tokyo University of Agriculture https://orcid.org/0000-0002-1621-9141

\section{Toshio Moriya}

High Energy Accelerator Research Organization https://orcid.org/0000-0001-7226-5487

\section{Naruhiko Adachi}

High Energy Accelerator Research Organization

\section{Yasuhiro Kamei}

National Institute for Basic Biology https://orcid.org/0000-0001-6382-1365

\section{Yasuhiro Kashino}

University of Hyogo https://orcid.org/0000-0002-4278-9359

\section{Sakae Kudoh}

National Institute of Polar Research

\section{Hiroyuki Koike}

Chuo University

\section{Toshiya Senda}

KEK (High Energy Accelerator Research Organization) https://orcid.org/0000-0002-5153-928X

\section{Article}

Keywords: Prasiola crispa, Pc-frLHC, photosynthesis

Posted Date: August 5th, 2021

DOI: https://doi.org/10.21203/rs.3.rs-701485/v1 
License: (c) (i) This work is licensed under a Creative Commons Attribution 4.0 International License. Read Full License 
2 Uphill energy transfer mechanism for photosynthesis in the 3 Antarctic alga

4

Makiko Kosugi ${ }^{1,2,3 *}$, Masato Kawasaki ${ }^{4,5 *}$, Yutaka Shibata ${ }^{6 *}$, Kojiro Hara ${ }^{7}$, Shinichi Takaichi $^{8}$, Toshio Moriya ${ }^{4}$, Naruhiko Adachi ${ }^{4}$, Yasuhiro Kamei ${ }^{9}{ }^{10}$, Yasuhiro Kashino ${ }^{11}$, Sakae Kudoh ${ }^{12,13}$, Hiroyuki Koike ${ }^{3}$, Toshiya Senda ${ }^{4,5,14 *}$

${ }^{1}$ Astrobiology Center, National Institutes of Natural Science, 2-21-1 Osawa, Mitaka, Tokyo 1818588, Japan.

${ }^{2}$ National Astronomical Observatory of Japan, 2-21-1 Osawa, Mitaka, Tokyo 181-8588, Japan

${ }^{3}$ Department of Biological Sciences, Faculty of Science and Engineering, Chuo University, 1-1327 Kasuga, Bunkyo-ku, Tokyo 112-8551, Japan.

${ }^{4}$ Structural Biology Research Center, Photon Factory, Institute of Materials Structure Science, High Energy Accelerator Research Organization (KEK), 1-1 Oho, Tsukuba, Ibaraki 305-0801, Japan.

${ }^{5}$ Department of Materials Structure Science, School of High Energy Accelerator Science, The Graduate University of Advanced Studies (Soken-dai), 1-1 Oho, Tsukuba, Ibaraki 305-0801, Japan.

${ }^{6}$ Department of Chemistry, Graduate School of Science, Tohoku University, 6-3 Aza Aoba Aramaki, Aoba-ku, Sendai 980-8578, Japan.

${ }^{7}$ Department of Biological Production, Akita Prefectural University, 241-438 Kaidobata-nishi, Shimoshinjo-nakano, Akita, 010-0195, Japan.

${ }^{8}$ Department of Molecular Microbiology, Tokyo University of Agriculture, 1-1 Sakuragaoka, Setagaya-ku, Tokyo 156-8502, Japan.

${ }^{9}$ National Institute for Basic Biology, National Institutes of Natural Sciences, 38 Nishigonaka, Myodaiji, Okazaki, Aichi 444-8585, Japan.

${ }^{10}$ Department of Basic Biology, School of Life Sciences, SOKENDAI (The Graduate University for Advanced Studies), 38 Nishigonaka, Myodaiji, Okazaki, Aichi 444-8585, Japan.

${ }^{11}$ Graduate School of Science, University of Hyogo, 3-2-1 Kohto, Kamigohri, Ako-gun, Hyogo 678-1297, Japan.

${ }^{12}$ National Institute of Polar Research, Research Organization of Information and Systems, 10-3 Midori-cho, Tachikawa, Tokyo 190-8518, Japan.

${ }^{13}$ Department of Polar Science, School of Multidisciplinary Science, SOKENDAI (The Graduate University for Advanced Studies), 10-3 Midori-cho, Tachikawa, Tokyo 190-8518, Japan.

${ }^{14}$ Faculty of Pure and Applied Sciences, University of Tsukuba, 1-1-1 Tennodai, Tsukuba, Ibaraki 
1 305-8572, Japan

2

$3 \quad$ "These authors contributed equally to this work.

4

5 *Correspondence and requests for materials should be addressed to M. Kosugi

$6 \quad$ (kosugi@bio.chuo-u.ac.jp), $\quad$ Y. $\quad$ Shibata $\quad$ (shibata@m.tohoku.ac.jp), and $\quad$ T. $\quad$ Senda

7 (toshiya.senda@kek.jp).

8

9 
3 Prasiola crispa, a major green alga in Antarctica, forms layered colonies for survival 4 under the severe terrestrial conditions of Antarctica, which include severe cold, drought, 5 and strong sunlight ${ }^{1,2}$. As a result of these conditions, the surface cells of $P$. crispa and 6 other Antarctic organisms face high risk of photodamage ${ }^{3}$. Cells of deeper layer escape 7 from photodamage at the sacrifice of photosynthetic active radiation except infrared. $P$. 8 crispa achieves effective photosynthesis by low energy far-red light for photosystem II 9 excitation with high efficiency similar to that of visible light. Here, we identified a farred light-harvesting complex of photosystem II in P. crispa, Pc-frLHC, and proposed a molecular mechanism of uphill excitation energy transfer based on its cryogenic electronmicroscopy structure. While Pc-frLHC is associated with photosystem II, it is evolutionarily related to the light-harvesting complex of photosystem I. Pc-frLHC forms a ring-shaped homo-undecamer in which all chlorophyll $a$ molecules are energetically connected and contains chlorophyll $a$ trimers. It seems that the trimers are longwavelength-absorbing chlorophylls for far-red light at $708 \mathrm{~nm}$, and further absorbance extension is accomplished by Davydov-splitting in dimeric chlorophylls. The chlorophyll network should enable a highly efficient entropy-driven uphill excitation energy transfer using far-red light up to $725 \mathrm{~nm}$. 
2 The capture of light energy and its transfer to photosynthetic reaction centers are the

3 primary photosynthetic processes that are performed in light-harvesting proteins with

4 photosynthetic pigments such as chlorophylls and carotenoids. To adapt to the various

5 light conditions under the various micro-environments on earth, pigment species and their

6 binding proteins became highly diversified during their evolution ${ }^{4,5}$.

Eukaryotic photoautotrophs commonly use chlorophyll $a(\mathrm{Chl} a)$-based lightharvesting complexes (LHCs). Subunits of LHCs typically have three transmembrane or photosystem II (PSII) ${ }^{7-9}$. Chl $a$-based PSII needs excitation energy corresponding to $680 \mathrm{~nm}$ light to split water molecules ${ }^{10}$. Since the light-harvesting chlorophyll protein complex of PSII (LHCII) contains Chl $a$ and the monomeric Chl $a$ shows a red absorption band (Qy band) around $670 \mathrm{~nm}$, monomeric Chl $a$ is efficiently used in downhill excitation energy transfer (EET) in LHCII together with a shorter wavelength absorbing $\mathrm{Chl}, \mathrm{Chl} b^{11}$. On the other hand, PSI requires excitation energy corresponding to $700 \mathrm{~nm}$ light $^{12}$, and the light-harvesting chlorophyll protein complexes of PSI (LHCI) use up to c.a. $720 \mathrm{~nm}$ light for PSI excitation ${ }^{13}$. LHCIs possess long-wavelength absorbing Chls (LWC), which consist of aggregates of Chl $a$ molecules ${ }^{14-16}$. LHCII, however, has little LWC.

Effective excitation of the eukaryotic PSII reaction center with far-red light is rather rare but has been reported in some algae ${ }^{17-19}$. The EET mechanism of the far-red light-capturing system is noteworthy from the perspective of energy yield. However, its mechanism and structural relevance have not been clarified. Furthermore, oxygenic photosynthesis with far-red light has also attracted the attention of astrobiology researchers. Since an abundance of far-red light characterizes the environments of most observable exoplanets revolving around red-dwarfs (low-mass stars) using nextgeneration telescopes, confirmation of the molecular mechanism of water-splitting photosynthesis by far-red light is a biological rationale of which oxygen in the atmosphere is a candidate of biosignature on the exoplanets ${ }^{20-23}$.

Prasiola crispa is one of the dominant green algae in Antarctica (Extended Data Fig. 1a $)^{24}$. P . crispa often makes large, layered colonies in terrestrial habitats. While cells beneath of the layered colony can escape from physical damage to their photosynthetic proteins induced by the triple stresses of severe cold, drought, and strong sunlight, the cost of protective strategy from the stresses causes a drastic decrease in net 
photosynthesis $^{1-3}$. That is, photosynthetically active radiation $(400-700 \mathrm{~nm})$ declines in the deep layers and only far-red light remains (Extended Data Fig. 1). Our analysis using cells of $P$. crispa suggested that LWC in P. crispa allows efficient PSII activation by farred light ${ }^{25}$. The LWC thus has a critical role in increasing photosynthetic productivity inside the layered colony (Extended Data Fig. 1b). Since excitation of PSII requires light of approximately $680 \mathrm{~nm}$, the far-red light harvesting system requires the uphill EET for PSII. Our earlier in vivo study revealed that $P$. crispa achieves highly efficient uphill EET with far-red light in the similar efficiency as downhill EET with visible light. To clarify the mechanism of the highly efficient uphill EET in P. crispa, we purified an LWCcontaining complex from $P$. crispa and analyzed it spectroscopically and structurally.

\section{Results}

\section{Identification of an LWC-binding protein}

We purified the LWC-binding protein complex from thylakoid membranes of P. crispa by sucrose density gradient centrifugation and anion exchange chromatography by monitoring the far-red absorption band (Fig. 1a). The purified LWC-binding protein complex was designated Pc-frLHC (Prasiola crispa far-red light-harvesting Chl-binding protein complex). In addition to a typical absorption band at $671 \mathrm{~nm}$ (Qy band), Pc-frLHC shows a large far-red absorption band at 706.5 together with fluorescence emission at around 713 nm (F713) at room temperature (Fig. 1b, Extended Data Fig. 2). Pc-frLHC is a homo-oligomer that is composed of a subunit of about $29 \mathrm{kDa}$ (Fig. 1c, right). While the exact molecular mass of Pc-frLHC was difficult to determine, the band of Pc-frLHC appeared at approximately the same position with the $700 \mathrm{kDa}$ marker protein on the native PAGE (Fig. 1c, left). The far-red Qy band can be fitted with two LWC components peaking at 708 and $725 \mathrm{~nm}$, with area ratios of 11:1 (Fig. 1d).

The amino acid sequence of the subunit of Pc-frLHC was deduced from cDNA sequences of the total mRNA libraries with the help of an internal amino acid sequence analysis of Pc-frLHC (Extended Data Figs. 3-4). The LHCII family proteins are known to be the only LHCs contributing to PSII excitation in the green lineage of photosynthetic eukaryotes (green algae and plants). Surprisingly, while Pc-frLHC delivers the excitation energy to PSII, Pc-frLHC is not a member of the LHCII family. The amino acid sequence of the Pc-frLHC subunit was classified into one of the LHCI groups (Extended Data Fig. 5). 


\section{Fluorescence measurement revealed two distinct Chl a pools}

The spectroscopic characteristics of the purified Pc-frLHC were analyzed with the timeresolved fluorescence spectrum at $273 \mathrm{~K}$. The excitation at $740 \mathrm{~nm}$ light generates fluorescence at $680 \mathrm{~nm}$ (F680) with a rise time of 25 ps (Fig. 2a). The longest time constant of the fluorescence decay was $2.2 \mathrm{~ns}$. This result suggests an efficient utilization of far-red light in Pc-frLHC realized by the uphill EET from LWC to bulk Chls. For further analysis, the time-resolved fluorescence at $80 \mathrm{~K}, 201 \mathrm{~K}$, and $273 \mathrm{~K}$ was measured with the excitation at $460 \mathrm{~nm}$ (Fig. 2b). The fluorescence emission peak of Pc-frLHC showed a temperature-dependent red shift from $710 \mathrm{~nm}(\mathrm{~F} 710)$ at room temperature to $730 \mathrm{~nm}(\mathrm{~F} 730)$ at $87 \mathrm{~K}$ (Fig. 2c) ${ }^{25}$. There are two possible explanations for the temperature-dependent red shift. The first is that the red shift arises due to temperaturedependent structural changes around the pigments that cause a change in the energy level of the Chl $a$ pool. However, our analysis supports a second explanation-namely, that there are two intrinsic $\mathrm{Chl} a$ pools, and lowering the temperature alters the equilibrium between them, causing the red-shifted emission.

The fluorescence decay associated spectra (FDAS) analysis of the time-resolved fluorescence spectra showed two FDAS components at $80 \mathrm{~K}$, one with a time constant of 250 ps peaking at $710 \mathrm{~nm}$ and the other with a time constant of $1.9 \mathrm{~ns}$ peaking at $730 \mathrm{~nm}$ (Figs 2d-f). This result supports the co-existence of the two independent pigment pools. The FDAS analysis also revealed a decay of F710 and rise of F730 with the same time constant of 250 ps (Fig. 2f), suggesting an energy-transfer from the Chl $a$ pool emitting F710 to that emitting F730 with a time constant of 250 ps.

The two pigment pools were further analyzed quantitatively based on the assumption that the red shift of the emission peak upon lowering the temperature is due to the shift of the equilibrium between the intrinsic Chl $a$ pools, F710 and F730. For this analysis, the fluorescence spectra at various temperatures were deconvoluted by the fitting of two Gaussian functions with peaks at $713 \mathrm{~nm}$ and $730 \mathrm{~nm}$ (Fig 2c). Based on the analysis using the Arrhenius equation, the ratio of the intrinsic emission intensity of F710 to that of F730 $\left(I_{\mathrm{F} 713} / I_{\mathrm{F} 730}\right)$ was estimated as approximately 12 (Fig $\left.\mathbf{2 g}\right)$. There are two possible models explaining the ratio of $I_{\mathrm{F} 713} / I_{\mathrm{F} 730}$. One attributes the intensity ratio to the different numbers of the $\mathrm{Chl} a$ molecules which constitute the pigment pools, and the other to the different oscillator strengths of the two emission bands. The second model assumes that the oscillator strength of F730 is 12-fold weakened due to its forbiddentransition nature. 
The tertiary structure of Pc-frLHC

3 To establish a structural basis for the spectroscopic characteristics of Pc-frLHC, the cryo4 EM structure of Pc-frLHC was determined at 3.13 A resolution (Supplementary Table

1, Extended Data Figs. 6, 7). Pc-frLHC is an undecamer with eleven-fold symmetry (Fig.

3a). Ring-shaped light-harvesting antennas have been reported only from LH1 and LH2 in anaerobic purple bacteria and IsiA in cyanobacteria ${ }^{26-28}$. Pc-frLHC is the first example of a ring-shaped eukaryotic LHC. While inter-subunit protein-protein interactions are tight at the stromal side, there are only limited inter-subunit interactions at the lumenal side. Due to the asymmetric interactions between the subunits, there is a large cavity at the interface of the two subunits. The cavity accommodates nine Chl molecules (Fig. 4). PSII in P. crispa is expected to be loosely bound outside of the Pc-frLHC ring structure, because no PSI/PSII subunits were detected by SDS-PAGE of the Pc-frLHC fraction. Moreover, PSII is too large to fit into the inner space of the ring.

The subunit of Pc-frLHC shares the basic fold of the typical LHC subunit with three transmembrane helices, which have been conventionally designated as helices-B, C, and A from the N-terminus (Fig. 3b). However, the subunit of Pc-frLHC has three unique structural features. First, the N-terminal loop region is significantly longer than its homologs (Extended Data Fig. 4). Second, the BC loop is relatively short and lacks a well-conserved helix-E. Third, the subunit of Pc-frLHC has a fourth transmembrane helix, helix-F, after helix-D, as observed in LHCI-type homologs of Pc-frLHC (Fig. 3b, Extended Data Fig. 4) ${ }^{29-32}$.

\section{Pigment arrangement in Pc-frLHC}

We found eleven Chls and two carotenoids in the subunit of Pc-frLHC (Fig. 3c, Extended Data Table 1). While high-performance liquid chromatography (HPLC) pigment analysis using C18-column suggested that at least one $\mathrm{Chl}$ in the subunit is Chl $b$ (Extended Data Fig. 8), it was impossible to distinguish Chl $a$ and $b$ in the current cryoEM map. We therefore modeled the eleven Chls as Chl $a$. The numbering of Chls in PcfrLHC was defined following the nomenclature of spinach $\mathrm{LHCII}^{33}$. All Chls but Chl708 in the subunit occupy similar positions to those of the corresponding Chls in spinach LHCII monomers. Chls form two layers; one is close to the stromal side (Chls601, 602, $603,609,610,611$, and 612) and the other is close to the lumenal side (Chls604, 613, and 614) (Fig. 3c). Chl708 is located between the two layers. 
Due to the inter-subunit interactions, seven consecutive Chls, Chl601-602-603609-611'-612'-610' on the stromal layer, are aligned from inside to outside of the undecameric ring at the stromal side, with Mg-Mg distances less than $15 \AA$ (Fig. 4a). The calculated exciton couplings (Extended data Table 2) suggest that two sets of seven consecutive Chls are energetically connected to each other through the Chl610Chl602/603 and Chl601'-Chl603/Chl609 interactions (Fig. 4a, Extended data Table 2). All Chls on the stromal layer form an energetically consecutive network throughout the undecameric ring. In addition, Chl708 energetically connects Chl603-609, Chl611', and the lumenal Chl613'-614' dimer and the stromal Chls.

\section{Trimeric and dimeric structure of Chls and their spectroscopic assignments}

Interestingly, Chl708, Chl609, and Chl603 form a trimer (Fig. 4a). Chl708 and Chl603 are located side-by-side, and Chl609 makes stacking $(\pi-\pi)$ interactions with Ch1708 and Chl603 (Fig. 4a). The trimer can be assigned to the main LWC component at $708 \mathrm{~nm}$ $\left(\mathrm{LWC}_{708}\right)$; the ratio of the numbers of the trimeric Chls to the total Chls in the subunit is $3: 11(=27 \%)$, which is consistent with the absorbance area ratio of $22 \%$ in the Qy band (Fig. 1d).

In addition to the Chl trimer, there is a Chl dimer, Chl613-614, which might be assigned as $\mathrm{LWC}_{725}$. The arrangement of the Chl613-614 dimer seems to allow Davydov splitting with the lower, forbidden-nature excited state ${ }^{34}$ (Supplementary Table 2; see the Methods section). The longest-wavelength absorbance at $725 \mathrm{~nm}$ and F730 seem to be assigned to the forbidden transition. The ratio of the absorbance band areas $\mathrm{LWC}_{708} / \mathrm{LWC}_{725}(22 / 2 \sim 11)$ (Fig. 1d) seems to correlate with the $I_{\mathrm{F} 713} / I_{\mathrm{F} 730}$ value $(\sim 12)$ calculated from the temperature-dependent fluorescence spectra (Fig. 2g).

Chls610, 611, and 612 are likely other functionally critical Chls, which probably serve collectively as the energy exit site. Since these Chls are located near the outside of the Pc-frLHC ring (Fig. 4a), they can interact with PSII on the outer surface of the PcfrLHC ring structure. Since Chls610, 611, and 612 are located just beside $\mathrm{LWC}_{708}$ of next subunit, the excited energy from $\mathrm{LWC}_{708}$ can be readily transferred to PSII.

\section{Discussion}

In this study, we demonstrated that Pc-frLHC in P. crispa can excite PSII with far-red light using uphill energy transfer from LWC to bulk Chls. Pc-frLHC has two LWCs, the main far-red light absorbing LWC, $\mathrm{LWC}_{708}$, and a further red-shifted $\mathrm{LWC}, \mathrm{LWC}_{725}$. 
Based on the spectroscopic and structural studies, we assigned $\mathrm{LWC}_{708}$ and $\mathrm{LWC}_{725}$ to the trimeric Chls603-609-708 and dimeric Chls613-614, respectively. The notable ringshaped structure of Pc-frLHC supports the formation of a large Chl network. This network seems to contribute to highly efficient energy transfer to PSII even using the uphill EET. Due to the uphill EET, only part of the absorbed energy can be used for PSII excitation theoretically. The thermal activation and entropy effect drive the uphill EET from $\mathrm{LWC}_{708}$ to bulk Chls. The Boltzmann distribution tells us that there is an approximately 5\% probability of finding an excited state in bulk Chls when the energy gap is $28 \mathrm{~nm}$ (from $708 \mathrm{~nm}$ to $680 \mathrm{~nm}$ ) at $278 \mathrm{~K}$. In addition, the entropy effect by the abundance ratio of the bulk Chls to $\mathrm{LWC}_{708}$ in Pc-frLHC (4:1) increases the probability fourfold, resulting in $20 \%$ probability in total. While the probability of the uphill EET of the entire Pc-frLHC was nearly the same as that of the monomer of the Pc-frLHC due to $\mathrm{LWC}_{708}$ and the bulk Chls having the same abundance ratios, the probability of energy transfer to PSII would increase significantly in the ring-shaped structure. The ring-shaped structure forms an energetically connected Chl network. Thus, eleven PSII-binding sites can play an equivalent role in the energy transfer to PSII, resulting in sufficient probability for the PSII excitation per $L H C$ with the far-red light (Fig. 4b). Theoretical calculation based on the current spectroscopic data and the cryo-EM structure suggests that the probability of EET from Pc-frLHC to a bound acceptor complex reaches almost $80 \%$ even if only one acceptor complex is bound. When five acceptor complexes are bound, $P_{\text {exit }}$ reaches $95 \%$ (Supplementary Note). In addition, we consider that $\mathrm{LWC}_{725}$ plays a role in another uphill EET. The action spectrum analysis of $P$. crispa's cells demonstrated that the highly efficient PSII excitation with far-red light was observed up to $750 \mathrm{~nm}^{25}$, suggesting that the reddest $\mathrm{LWC}_{725}$ can contribute to the energy transport to PSII by two-step uphill EET through $\mathrm{LWC}_{708}$ and the bulk Chls in Pc-frLHC.

In summary, Pc-frLHC with $\mathrm{LWC}_{725}$ and $\mathrm{LWC}_{708}$ can excite PSII by uphill EET using far-red light. The ratios of $\mathrm{LWC}_{725}, \mathrm{LWC}_{708}$, and bulk Chls are suitable for the highly efficient uphill EET. Moreover, the ring-shaped arrangement of the eleven subunits enables the formation of an energetically connected Chl network with eleven excited-energy exit sites, increasing the probability of energy transfer to PSII. Pc-frLHC is a well-designed energy transfer molecular machine using far-red light. Pc-frLHC contributes to the predominant growth of P. crispa in the Antarctic terrestrial habitat through the realization of efficient photosynthesis with a far-red light. 


\section{Main references}

1. Block, W. Terrestrial ecosystems: Antarctica. Polar Biol. 14, 293-300 (1994).

2. Perera-Castro, A. V., Flexas, J., González-Rodríguez, Á. M. \& Fernández-Marín, B. Photosynthesis on the edge: photoinhibition, desiccation and freezing tolerance of Antarctic bryophytes. Photosynth. Res. (2020) doi:10.1007/s11120-020-00785-0.

3. Kosugi, M. et al. A comparative study of wavelength-dependent photoinactivation in photosystem II of drought-tolerant photosynthetic organisms in Antarctica and the potential risks of photoinhibition in the habitat. Ann. Bot. 122, 1263-1278 (2018).

4. Mirkovic, T. et al. Light absorption and energy transfer in the antenna complexes of photosynthetic organisms. Chem. Rev. 117, 249-293 (2017).

5. Green, B. and Parson, W.W. Light-Harvesting Antennas in Photosynthesis. (Springer Netherlands, 2003). doi:10.1007/978-94-017-2087-8.

6. Kühlbrandt, W., Wang, D. N. \& Fujiyoshi, Y. Atomic model of plant light-harvesting complex by electron crystallography. Nature 367, 614-621 (1994).

7. Wei, X. et al. Structure of spinach photosystem II-LHCII supercomplex at $3.2 \AA$ resolution. Nature 534, 69-74 (2016).

8. Ben-Shem, A., Frolow, F. \& Nelson, N. Crystal structure of plant photosystem I. Nature 426, 630-635 (2003).

9. Pan, X. et al. Structure of the maize photosystem I supercomplex with lightharvesting complexes I and II. Science 360, 1109-1113 (2018).

10. Döring, G., Renger, G. \& Witt, H.T. Properties of the photoactive chlorophyll-a photosynthesis. Z. Naturforschung 24, 1139-1143 (1969).

11. Dau, H. \& Zaharieva, I. Principles, efficiency, and blueprint character of solar-energy conversion in photosynthetic water oxidation. Acc. Chem. Res. 42, 1861-1870 (2009).

12. KoK, B. Significance of $\mathrm{P} 700$ as an intermediate in photosynthesis. Proc. Int. Congr. Biochem. 6, 73-81 (1963). 
13. Joliot, P., Joliot, A. \& Kok, B. Analysis of the interactions between the two photosystems in isolated chloroplasts. Biochim. Biophys. Acta - Bioenerg. 153, 635652 (1968).

14. Morosinotto, T., Ballottari, M., Klimmek, F., Jansson, S. \& Bassi, R. The association of the antenna system to photosystem I in higher plants. Cooperative interactions stabilize the supramolecular complex and enhance red-shifted spectral forms. J. Biol. Chem. 280, 31050-31058 (2005).

15. Morosinotto, T., Mozzo, M., Bassi, R. \& Croce, R. Pigment-pigment interactions in Lhca4 antenna complex of higher plants photosystem I. J. Biol. Chem. 280, 2061220619 (2005).

16. Mozzo, M. et al. Functional analysis of photosystem I light-harvesting complexes (Lhca) gene products of Chlamydomonas reinhardtii. Biochim. Biophys. Acta Bioenerg. 1797, 212-221 (2010).

17. Wilhelm, C. \& Jakob, T. Uphill energy transfer from long-wavelength absorbing chlorophylls to PS II in Ostreobium sp. is functional in carbon assimilation. Photosynth. Res. 87, 323-329 (2006).

18. Kotabová, E. et al. Novel type of red-shifted chlorophyll $a$ antenna complex from Chromera velia. I. Physiological relevance and functional connection to photosystems. Biochim. Biophys. Acta - Bioenerg. 1837, 734-743 (2014).

19. Wolf, B. M. et al. Characterization of a newly isolated freshwater Eustigmatophyte alga capable of utilizing far-red light as its sole light source. Photosynth. Res. 135, 177-189 (2018).

20. Brandt, T. D. \& Spiegel, D. S. Prospects for detecting oxygen, water, and chlorophyll on an exo-Earth. Proc. Natl. Acad. Sci. 111, 13278-13283 (2014).

21. Cowan, N. B. et al. Characterizing transiting planet atmospheres through 2025. Publ. Astron. Soc. Pac. 127, 311-327 (2015). 
22. Meadows, V. S. Reflections on $\mathrm{O}_{2}$ as a biosignature in exoplanetary atmospheres. Astrobiology 17, 1022-1052 (2017).

23. Meadows, V. S. et al. Exoplanet biosignatures: understanding oxygen as a biosignature in the context of its environment. Astrobiology 18, 630-662 (2018).

24. Broady, P. A. Diversity, distribution and dispersal of Antarctic terrestrial algae. Biodivers. Conserv. 5, 1307-1335 (1996).

25. Kosugi, M. et al. Red-shifted chlorophyll $a$ bands allow uphill energy transfer to photosystem II reaction centers in an aerial green alga, Prasiola crispa, harvested in Antarctica. Biochim. Biophys. Acta - Bioenerg. 1861, 148139 (2020).

26. Prince, S. M. et al. Apoprotein structure in the LH2 complex from Rhodopseudomonas acidophila strain 10050: modular assembly and protein pigment interactions. J. Mol. Biol. 268, 412-423 (1997).

27. Roszak, A. W. et al. Crystal structure of the RC-LH1 core complex from Rhodopseudomonas palustris. Science 302, 1969-1972 (2003).

28. Toporik, H., Li, J., Williams, D., Chiu, P.-L. \& Mazor, Y. The structure of the stressinduced photosystem I-IsiA antenna supercomplex. Nat. Struct. Mol. Biol. 26, 443449 (2019).

29. Su, X. et al. Antenna arrangement and energy transfer pathways of a green algal photosystem-I-LHCI supercomplex. Nat. Plants 5, 273-281 (2019).

30. Suga, M. et al. Structure of the green algal photosystem I supercomplex with a decameric light-harvesting complex I. Nat. Plants 5, 626-636 (2019).

31. Caspy, I. et al. Structure and energy transfer pathways of the Dunaliella Salina photosystem I supercomplex. Biochim. Biophys. Acta - Bioenerg. 1861, 148253 (2020).

32. Qin, X. et al. Structure of a green algal photosystem I in complex with a large number of light-harvesting complex I subunits. Nat. Plants 5, 263-272 (2019). 
1 33. Liu, Z. et al. Crystal structure of spinach major light-harvesting complex at $2.72 \AA$ $2 \quad$ resolution. Nature 428, 287-292 (2004).

3 34. Hestand, N. J. \& Spano, F. C. Expanded theory of H- and J-molecular aggregates: the 4 effects of vibronic coupling and intermolecular charge transfer. Chem. Rev. 118, $5 \quad$ 7069-7163 (2018).

6 

arbitrary unit.

Figure 1 Purification of Pc-frLHC. a: The purification scheme of Pc-frLHC and other photosynthetic proteins from P. crispa's thylakoid membranes. b: Absorbance spectra (solid line) and fluorescence spectrum (dotted line) of the thylakoids (red) and Pc-frLHC (black) measured at room temperature. c: The hrCN-PAGE (left) and SDS-PAGE (right) analyses of thylakoids (lane 1), PSII-LHCII (lane 2), LHCII (lane 3), PSI-LHCI (lane 4) and Pc-frLHC (lane 5). d: The fitting analysis of the absorbance spectra of purified PcfrLHC at room temperature. The peak wavelengths of each component were estimated by the second and fourth derivative of the absorbance spectrum, and fitting analysis with Gaussian functions was performed by Magic plot 2.7.2 (Magicplot Systems). a.u.:

Figure 2 Spectroscopic analysis of Pc-frLHC. a,b: (a) Fluorescence time profiles of Pc-frLHC excited at $740 \mathrm{~nm}$ and monitored at $680 \mathrm{~nm}$ (green) observed at $273 \mathrm{~K}$ and (b) those excited at $460 \mathrm{~nm}$ and monitored at $680 \mathrm{~nm}$ (green), $710 \mathrm{~nm}$ (orange), and $740 \mathrm{~nm}$ (red) and observed at $80 \mathrm{~K}$. Blue curves show the fitting curves to the sum of three exponential components convolved with the instrumental response function shown by red circles in (a). c: Temperature dependence of the fluorescence spectrum of Pc-frLHC excited at $460 \mathrm{~nm}$. The blue curves are the fitting curves to the sum of two Gaussian functions (the filled green and orange curves). d-f: Fluorescence-decay-associated spectra of Pc-frLHC excited at $460 \mathrm{~nm}$ and observed at $273 \mathrm{~K}$ (d), $201 \mathrm{~K}$ (e), and $80 \mathrm{~K}$ (f). g: Analysis of the ratio Areaf713/Areaf730 using the Arrhenius equation. The blue line is the fitting according to equation S1 (see Methods) with the energy gap fixed to 318 $\mathrm{cm}^{-1}$. a.u.: arbitrary unit.

Figure 3 Overall cryo-EM structure of Pc-frLHC. a: Top (upper panel) and side views (lower panel) are shown. Each subunit is shown in a different color. b: Top and side views of the subunit of Pc-frLHC in rainbow colors from the N-terminus in blue to C-terminus in red. c: Chlorophyll $a$ arrangement in Pc-frLHC (the left panel). Each chlorophyll is colored in the same color as the corresponding subunit in (a). The right panel shows the arrangement of chlorophyll $a$ in the subunit. Chlorophylls on the stromal and lumenal sides are shown in green and cyan. Chl708 is shown in purple.

Figure 4 The chlorophyll network in Pc-frLHC. a: Connections among chlorophyll $a$ 
1 in Pc-frLHC. Energetically connected stromal chlorophylls (including Chl708) are linked

2 by dotted lines (Extended data Table 2). The dotted lines are colored based on the values

3 of excitonic couplings (EC): $E C>60$ in red, $60 \geq E C>30$ in orange, and $30 \geq E C>10$

4 in gray. Chlorophylls on the stromal and lumenal sides are shown in green and cyan,

5 respectively. Chl708 is shown in purple. While Chls613, 614 are energetically connected

6 to Chls708, 612 and 601, they are partly shown in this figure for clarity. b: Schematic

7 drawing of excited energy flows in Pc-frLHC. Pc-frLHC absorbs far red light using

$8 \quad$ LWCs and distributes its energy to PSII by uphill excitation energy transfer.

9

10 


\section{Methods}

\section{Protein purification and characterization}

Thylakoid membranes were prepared from thalli of $P$. crispa harvested from Antarctica as described in our recent report ${ }^{25}$. For purification of Pc-frLHC, the thylakoids were diluted with Buffer-A (25 mM MES, $1 \mathrm{M}$ betaine, $10 \mathrm{mM} \mathrm{MgCl}$, $5 \mathrm{mM}$ EDTA, $12.5 \%$ glycerol) and solubilized at $0.5 \mathrm{mg} \mathrm{Chl} \mathrm{ml}^{-1}$ with $1 \%$ dodecyl- $\beta$-D-maltoside ( $\beta$-DDM) on ice for $20 \mathrm{~min}$. The solubilized sample was centrifuged with an Optima ${ }^{\mathrm{TM}}$ TLX and TLA-110 rotor (Beckman Coulter, Brea, CA) at 20,000 $\times g$ for $20 \mathrm{~min}$. The supernatant was fractionated by sucrose density gradient (SGD) centrifugation at $267,000 \times g$, at $4{ }^{\circ} \mathrm{C}$ for $16 \mathrm{~h}$. The middle green band containing PSI-LHCI and Pc-frLHC was collected and diluted ten times with Buffer-B (25 mM MES, 1M betaine, $0.03 \% \beta$-DDM). The upper green band and lowest light green part were collected as LHCII and PSII-LHCII fractions, respectively. The diluted fraction was adsorbed on a diethylaminoethyl cellulose column (Whatman DE52), and the PSI-LHCI fraction was washed out with $150 \mathrm{mM} \mathrm{NaCl}$ containing Buffer-B. The Pc-frLHC fraction was eluted with $250 \mathrm{mM} \mathrm{NaCl}$ containing Buffer-B, diluted five times with Buffer-B and precipitated by centrifugation at 417,000 $\times g$ at $4^{\circ} \mathrm{C}$ for $2 \mathrm{~h}$. For fluorescence measurements, pigment analysis, and cryo-EM analysis, the concentrated fraction was fractionated again by SDG centrifugation and the lowest dark green band was collected. Sucrose was removed by dilution with Buffer-B and ultracentrifugation cycles.

The purified Pc-frLHC fraction was characterized by absorbance and fluorescence spectra measurements, high resolution clear native ( $\mathrm{hrCN}$ )-polyacrylamide gel electrophoresis (PAGE), SDS-PAGE, and HPLC. hrCN-PAGE and SDS-PAGE were performed according to the previously described protocols $\mathrm{s}^{35,36}$. The absorption and fluorescence spectra at room temperature were measured with an MPS-2450 (Shimadzu, Kyoto, Japan) and RF-6000 fluorescence spectrometer (Shimadzu, Kyoto, Japan), respectively, as described in our recent report ${ }^{25}$. Pigment analysis was performed by HPLC as described by Takaichi et al. ${ }^{37}$ The purified Pc-frLHC fraction was injected directly into the HPLC system and the absorbance at $440 \mathrm{~nm}$ was monitored.

\section{Amino acid sequence analysis}

The Pc-frLHC protein was separated with SDS-PAGE, and the gel was stained with CBB solution (0.1\% CBB-R, $10 \%$ acetic acid and 50\% methanol) and destained with $25 \%$ 
methanol containing $10 \%$ acetic acid. The band at $29 \mathrm{kDa}$ was cut out and put in a microtube for lysyl endopeptidase treatment. The peptidase treatment was performed according to an earlier described protocol ${ }^{38}$. The fragmented peptides were separated by SDS-PAGE and electrophoretically blotted onto a PVDF membrane. The N-terminal amino acid sequences of four peptides were determined with a Procise 492cLC (Applied Biosystems, Carlsbad, CA). The peptide sequence data were deposited in the UniProt Knowledgebase under the accession number COHLU5. We searched for the most probable cDNA of Pc-frLHC by using the determined internal peptide sequences from the total mRNA libraries of $P$. crispa (BioProject ID: PRJNA329112) ${ }^{39}$. The cDNA sequence of Pc-frLHC gene was submitted to Third PArty data (TPA) of the DDBJ/EMBL/GenBank databases and was assigned the accession numbers TPA: BR001753.

\section{Cryo-EM sample preparation and data collection}

For cryo-grid preparation, $3 \mu \mathrm{l}$ of $6 \mathrm{mg}_{\text {protein }} \mathrm{ml}^{-1} \mathrm{Pc}-\mathrm{frLHC}$ (25 mM MES (pH 6.5), $0.015 \% \beta$-DDM, and $0.5 \mathrm{M}$ betain) was applied onto a holey carbon grid (Quantifoil, $\mathrm{Cu}$, $\mathrm{R} 1.2 / 1.3,300$ mesh), which was rendered hydrophilic by a $30 \mathrm{~s}$ glow-discharge in air (11 mA current) with a PIB-10 ion bombarder (Vacuum Device, Ibaraki, Japan). The grid was blotted for $5 \mathrm{~s}$ with a blot force of 25 at $18^{\circ} \mathrm{C}$ and $100 \%$ humidity. Then the grid was flash-frozen in liquid ethane using Vitrobot Mark IV (Thermo Fisher Scientific). For automated data collection, 1,555 micrographs were acquired with a Talos Arctica (Thermo Fisher Scientific) microscope operating at $200 \mathrm{kV}$ in the nanoprobe mode using EPU software. The movie micrographs were collected by a $4 \mathrm{k} \times 4 \mathrm{k}$ Falcon $3 \mathrm{EC}$ direct electron detector (electron counting mode) at a nominal magnification of 92,000 (1.13 $\AA /$ pixel). Fifty movie frames were recorded at an exposure of $1.00 \mathrm{e}^{-} / \AA^{2}$ frame, corresponding to a total exposure of $50 \mathrm{e}^{-} / \AA^{2}$. The defocus steps used were 1.0, 1.5, 2.0, 2.5 , and $3.0 \mu \mathrm{m}$.

\section{Cryo-EM data processing}

The cryo-EM data processing is summarized in Extended data Fig. 7. First, the movie frames were aligned, dose-weighted, and averaged using an algorithm implemented RELION $3^{40}$ on 5 x 5 tiled frames with a B-factor of 200 . The non-weighted movie sums were used for Contrast Transfer Function (CTF) estimation with the Gctf program ${ }^{41}$, 
while the dose-weighted sums were used for all subsequent steps of image processing. Particles were picked fully automatically using SPHIRE crYOLO ${ }^{42,43}$ with a generalized model using a selection threshold of 0.02. The subsequent processes, namely, 2D classification, ab initio reconstruction, 3D classification, 3D refinement, CTF refinement, and Bayesian polishing, were performed by using RELION $3^{40}$.

A stack of 696,095 particle images was extracted from the 1,555 dose-weighted sum micrographs while rescaling to a box of 144 pixels in size at $3.39 \AA /$ pixel and was subjected to 2D classification (200 expected classes). 195,767 particles corresponding to the best eleven classes, which displayed secondary-structural elements and multiple views of Pc-frLHC, were selected for ab initio map reconstruction. Two classes were clearly top views of a ring structure composed of eleven subunits with rotational symmetry (Extended data Fig. 7c). Therefore, C11 symmetry was imposed on the generated $a b$ initio map and used as an initial reference map for the 3D classification. 654,477 particles from 32 classes after 2D classification were selected with more relaxed criteria for the subsequent 3D classification (4 expected classes). The 3D volume and 185,680 particles of the $3 \mathrm{D}$ class with the highest resolution were used as the inputs of the subsequent 3D refinements with C11 symmetry. The refined volume and particle images were rescaled to a box of 432 pixels in size at $1.13 \AA$ /pixel. The particle images which became duplicated as the result of alignments were excluded. 160,157 selected particles were 3D auto-refined (C11 symmetry, with a mask diameter of $280 \AA$ ) twice (the 1 st run was without, and the 2 nd run was with a soft-edged 3D mask). One cycle of Bayesian polishing and CTF refinement was done, followed by $3 \mathrm{D}$ refinement with a softedged 3D mask (C11 symmetry, with a mask diameter of $360 \AA$ ) after each Bayesian polishing and CTF refinement step.

Then, no-alignment 3D classification was conducted (C11 symmetry, two expected classes, $\mathrm{T}=4$ ) with a soft-edged 3D mask, and 99,510 particles were selected by choosing the best 3D class. The last 3D refinement (C11 symmetry, with mask diameter $460 \AA$ ) with a soft-edged 3D mask generated the result at $3.13 \AA$ resolution. The gold standard FSC resolution with a 0.143 criterion $^{44}$ was used as the global resolution estimation. The local resolution was estimated using an algorithm implemented on RELION3. UCSF Chimera ${ }^{45}$ was used for the visualization.

\section{Model building, refinement, and validation}

The initial model was built using Map_to_Model ${ }^{46}$ in PHENIX software. The model was 
manually corrected by $\operatorname{Coot}^{47}$, followed by Real-space Refinement ${ }^{48}$ in PHENIX. The model was refined by multiple cycles of manual modifications in Coot and Real-space Refinement in PHENIX. NCS restraints were used for the automatic refinement. Validation of the refined model was carried out using MolProbity ${ }^{49}$ in PHENIX. UCSF Chimera and PyMOL (Schrödinger, LLC) were used for visualization.

\section{Fluorescence measurement}

The time-resolved fluorescence spectra were measured by the streak scope (C1067; Hamamatsu Photonics Inc., Hamamatsu, Japan). The magic angle configuration was used. 1 For the measurements with the excitations at $740 \mathrm{~nm}$, a femtosecond Ti:S laser (MAITAI; 2 Spectra-Physics, Santa Clara, CA) was used as the excitation source. A short-pass filter with a cut-off wavelength of $700 \mathrm{~nm}$ (FESH0700; Thorlabs, Newton, NJ) was set before the detector to block the excitation laser. For the measurements with the excitation at 460 $\mathrm{nm}$, we used the second-harmonics pulse of the Ti:S laser generated by a barium borate (BBO) crystal and a long-pass colored glass filter with a cut-off of $480 \mathrm{~nm}$. The 7 instrumental response function was determined by measuring a standard sample (aqueous 8 solution of Malachite Green) which was known to have a very short fluorescence 9 lifetime $^{50}$. For the steady-state fluorescence spectral measurements, a conventional fluorometer (F4500; Hitachi) was used.

1 The sample solution was set in the copper sample holder of the home-built Dewar. 2 For the measurements at $273 \mathrm{~K}, 201 \mathrm{~K}$, and $80 \mathrm{~K}$, the Dewar was filled with ice in a liquid 3 water bath, dry ice in a liquid ethanol bath, and liquid nitrogen as the cooling medium, 4 respectively. The Pc-frLHC solution in the buffer was mixed with a two-fold volume of 5 glycerol to maintain transparency of the solution, except for the measurement of the time6 resolved fluorescence at $273 \mathrm{~K}$.

\section{Estimation of the excitonic coupling}

We calculated the excitonic coupling between molecules A and B using the following equation:

$$
V_{\mathrm{A}, \mathrm{B}}=\frac{1}{\epsilon} \sum_{I=1}^{N} \sum_{J=1}^{N} \frac{q_{I}^{\mathrm{A}} q_{J}^{\mathrm{B}}}{r_{I J}}
$$

Here, $q_{I}^{\mathrm{A} / \mathrm{B}}$ is the atomic transition charges of the $I$-th atom in the molecule $\mathrm{A} / \mathrm{B}$, and $r_{I J}$ is the distance between the $I$-th atom in A and $J$-th atom in B. $\epsilon$ (here set to 2.0) is the 
dielectric constant of the protein matrix which shields the electrostatic interaction. We used the values of the atomic transition charges reported previously ${ }^{51}$, which were obtained by quantum chemical calculations (Hartree-Fock and configuration interaction with single excitations) of the ground and excited states of Chl $a$ in a vacuum. The results of the calculations are shown in Extended data Table 2.

\section{Analysis using the Arrhenius equation}

Figure $2 \mathrm{c}$ shows a slight but significant broadening of the emission band in the intermediate temperature region from $144 \mathrm{~K}$ to $183 \mathrm{~K}$, suggesting the overlap of two emission bands. The blue curves in Fig. $2 c$ are the fitting curves to the sum of two Gaussian functions with peaks at $713 \mathrm{~nm}$ and $730 \mathrm{~nm}$. The fitting was done with a constraint that the peak position of each band takes the same value for the data at every temperature. Figure $2 \mathrm{~g}$ shows the Arrhenius plot ${ }^{52}$ of the ratio of the band areas of the $713 \mathrm{~nm}$ band with respect to that of the $730 \mathrm{~nm}$. The blue straight line is the fitting according to the equation

$$
\frac{\text { Area }_{\mathrm{F} 713}}{\text { Area }_{\mathrm{F} 730}}=\frac{I_{\mathrm{F} 713}}{I_{\mathrm{F} 730}} \exp \left[-\frac{\Delta E}{k_{B} T}\right]
$$

Here, $k_{B}$ is Boltzmann's constant. $I_{\mathrm{F} 713 / \mathrm{F} 730}$ and $\Delta E$ are the intrinsic emission intensity of the Chl $a$ pool emitting the F713/F730 fluorescence band and the energy gap between the excited states of the two pigment pools, respectively. The fitting line is obtained with the value of $\Delta \mathrm{E}$ fixed to $318 \mathrm{~cm}^{-1}(=458 \mathrm{~K})$, which is calculated from the energy difference of the two peak wavelengths (Fig. 2c). Finally, we could estimate the value of $I_{\text {F713/F730 }}$ as approximately 12, suggesting that the F713 emission is intrinsically about 12-fold brighter than the F730 one.

\section{Davydov splitting of the Chl613-614 dimer}

Extended data Table 2 reveals several Chl pairs with significantly strong excitonic couplings. These Chl pairs are considered to form mixed excited states delocalized over the two molecules. These two mixed excited states are energetically separated with an energy known as the Davydov splitting, which is approximated by the double of the excitonic couplings. This effect is thought to be one of the major causes of the red shift of LWC. Since $\mathrm{LWC}_{725}$ was predicted to have a low oscillator strength, its potential candidate is assigned to a $\mathrm{Chl}$ pair that has the lower, forbidden-transition-nature excited state. A famous example of such a lower, forbidden-nature excited state is an H-type molecular aggregate, in which the transition dipole moments are aligned in a side-by-side 
configuration $^{34}$. When the excitonic coupling between molecules A and B is positive, the

2 transition dipole moment of the lower excited state can be approximated by

$$
\vec{\mu}_{-} \approx\left(\vec{\mu}_{\mathrm{A}}-\vec{\mu}_{\mathrm{B}}\right) / \sqrt{2},
$$

while when the coupling is negative it can be approximated by

$$
\vec{\mu}_{-} \approx\left(\vec{\mu}_{\mathrm{A}}+\vec{\mu}_{\mathrm{B}}\right) / \sqrt{2}
$$

Here, $\vec{\mu}_{\mathrm{A} / \mathrm{B}}$ is the transition dipole moment of the molecule A/B. The transition dipole moment of a Chl $a$ is known to be aligned nearly parallel to the vector from NB to ND (the nomenclature of the nitrogen atoms according to the PDB format) ${ }^{53}$. As shown in Supplementary Table 2, we found that only the Chl613-614 pair fulfills the above criterion. A simple calculation assuming the same excitation energy for Chl613 and 614 resulted in a transition dipole moment of the lower excited state of the Ch1613-614 pair that was reduced by approximately $33 \%$ from that of the monomeric $\mathrm{Chl} a$.

\section{Kinetic analysis of the EET}

The fluorescence decay curves at various wavelengths were fitted to sum of exponential functions:

$$
F_{\lambda}(t)=\left[\sum_{i=1}^{n} A_{i} \exp \left(-\frac{t}{\tau_{i}}\right) H(t)\right] \otimes I(t)
$$

Here, $I(t)$ is the instrumental response functions (Fig. 2a open circles), the symbol $\otimes$ indicates convolution, and $H(t)$ is the Heaviside step function. The fitting was done under a constraint that the time constants take the same values for every monitoring wavelength (global fitting). Three exponential components were found to be sufficient to fit the data. The pre-exponential factors plotted against the monitoring wavelength are called fluorescence decay-associated spectra (FDAS $)^{54}$ and are shown in Figs. 2d,e and f. Positive and negative signs in FDAS mean that the fluorescence at that wavelength has components that decay and rise with the time constant of the FDAS, respectively. Thus, the energy transfer is reflected in the profile of FDAS having positive and negative signs on the shorter and longer wavelength sides, respectively. 


\section{Method references}

35. Ikeuchi, M. \& Inoue, Y. A new 4.8-kDa polypeptide intrinsic to the PS II reaction center, as revealed by modified SDS-PAGE with improved resolution of lowmolecular-weight proteins. Plant Cell Physiol. 29, 1233-1239 (1988).

36. Wittig, I., Karas, M. \& Schägger, H. High resolution clear native electrophoresis for in-gel functional assays and fluorescence studies of membrane protein complexes. Mol. Cell. Proteomics 6, 1215-1225 (2007).

37. Takaichi, S. et al. Direct injection of pigment-protein complexes and membrane fragments suspended in water from phototrophs to $\mathrm{C}_{18}$ HPLC. Photosynth. Res. 144, 101-107 (2020).

38. Rosenfeld, J., Capdevielle, J., Guillemot, J. C. \& Ferrara, P. In-gel digestion of proteins for internal sequence analysis after one- or two-dimensional gel electrophoresis. Anal. Biochem. 203, 173-179 (1992).

39. Carvalho, E. L. et al. De novo assembly and annotation of the Antarctic alga Prasiola crispa transcriptome. Front. Mol. Biosci. 4, 89 (2018).

40. Zivanov, J. et al. New tools for automated high-resolution cryo-EM structure determination in RELION-3. eLife 7, e42166 (2018).

41. Zhang, K. Gctf: Real-time CTF determination and correction. J. Struct. Biol. 193, 112 (2016).

42. Moriya, T. et al. High-resolution single particle analysis from electron cryomicroscopy images using SPHIRE. J. Vis. Exp. 55448 (2017) doi:10.3791/55448.

43. Wagner, T. et al. SPHIRE-crYOLO is a fast and accurate fully automated particle picker for cryo-EM. Commun. Biol. 2, 218 (2019).

44. Rosenthal, P. B. \& Henderson, R. Optimal determination of particle orientation, absolute hand, and contrast loss in single-particle electron cryomicroscopy. J. Mol. Biol. 333, 721-745 (2003). 
45. Pettersen, E. F. et al. UCSF Chimera-a visualization system for exploratory research and analysis. J. Comput. Chem. 25, 1605-1612 (2004).

46. Terwilliger, T. C., Adams, P. D., Afonine, P. V. \& Sobolev, O. V. A fully automatic method yielding initial models from high-resolution cryo-electron microscopy maps. Nat. Methods 15, 905-908 (2018).

47. Emsley, P. \& Cowtan, K. Coot : model-building tools for molecular graphics. Acta Crystallogr. D Biol. Crystallogr. 60, 2126-2132 (2004).

48. Afonine, P. V. et al. Real-space refinement in PHENIX for cryo-EM and crystallography. Acta Crystallogr. Sect. Struct. Biol. 74, 531-544 (2018).

49. Williams, C. J. et al. MolProbity: More and better reference data for improved allatom structure validation. Protein Sci. 27, 293-315 (2018).

50. Yoshizawa, M., Suzuki, K., Kubo, A. \& Saikan, S. Femtosecond study of $\mathrm{S}_{2}$ fluorescence in malachite green in solutions. Chem. Phys. Lett. 290, 43-48 (1998).

51. Madjet, M. E., Abdurahman, A. \& Renger, T. Intermolecular coulomb couplings from ab initio electrostatic potentials: application to optical transitions of strongly coupled pigments in photosynthetic antennae and reaction centers. J. Phys. Chem. B 110, 17268-17281 (2006).

52. Pålsson, L.O. et al. Energy transfer and charge separation in photosystem I: P700 oxidation upon selective excitation of the long-wavelength antenna chlorophylls of Synechococcus elongatus. Biophys. J. 74, 2611-2622 (1998).

53. Linke, M., Lauer, A., von Haimberger, T., Zacarias, A. \& Heyne, K. Threedimensional orientation of the $\mathrm{Q}_{\mathrm{y}}$ electronic transition dipole moment within the chlorophyll a molecule determined by femtosecond polarization resolved VIS pump-IR probe spectroscopy. J. Am. Chem. Soc. 130, 14904-14905 (2008).

54. Knutson, J. R., Walbridge, D. G. \& Brand, L. Decay-associated fluorescence spectra and the heterogeneous emission of alcohol dehydrogenase. Biochemistry 21, 4671- 
144679 (1982).

2

3 


\section{Acknowledgements}

We thank Ms. Yumiko Makino of National Institute for Basic Biology (NIBB) for supporting of N-terminal amino acid sequence analysis. We also thank Dr. Hiroshi Imai of Osaka University and Dr. Keisuke Kawakami of RIKEN SPring-8 Center for technical advice about sample preparation for cryo-EM analysis. We thank Dr. Kotaro Koiwai of KEK for help with 3D model building. This work was supported by Grants-in-Aid from the Japanese Ministry of Education, Culture, Sports, Science, and Technology (grant number 17K19431 to M.Ko., 19H03187 to Y.S.). This work also supported by the Sumitomo Foundation (grant number 151376 to M.Ko), National Institute of Polar Research through General Collaboration Project (no 31-29 to Y.Kas) and the NIBB Collaborative Research Program (no 17-702, 18-506, 19-704 to M.Ko). This work was partly supported by the Platform Project for Supporting Drug Discovery and Life Science Research (Basis for Supporting Innovative Drug Discovery and Life Science Research (BINDS)) from the Japan Agency for Medical Research and Development (AMED) under Grant Number JP20am0101071 to TS (supporting no. 1649). The cryo-EM data were collected at the cryo-EM facility in KEK (Ibaraki, Japan).

\section{Author contributions}

M.Ko. conceived and designed the experiments. M.Ko. and S.K. collected the Prasiola crispa sample from Antarctica. M.Ko. purified the samples. M.Ko., S.T., and Y.Kas. performed biochemical analyses. M.Ko., Y.Kam., and K.H. performed primary sequence analysis. M.Ko., M.Ka., N.A. prepared the cryo-EM samples and collected data. M.Ka. and T.M. processed the cryo-EM data. M.Ka. performed model building, refinement, and validation of the cryo-EM structure. M.Ko., and Y.S. performed the spectroscopic experiments and analysis. M.Ko., Y.S., and T.S. analyzed data and interpreted results with contributions from H.K. and wrote the manuscript.

Competing interests: The authors declare no competing financial interests.

\section{Data availability}

Cryo-EM map of Pc-frLHC is deposited in the Electron Microscopy Data Bank under accession codes EMD-31486. Structure coordinate related to the cryo-EM map is deposited at the Protein Data Bank with accession code 7F78.

\section{Additional information}


Correspondence and requests for materials should be addressed to M.Ko, Y.S., or

3 T.S.

5 Extended data figure/ table legends

6 Extended Data Figure 1| Prasiola crispa in Antarctica, light conditions of the $\boldsymbol{P}$. crispa colony, and spectroscopic properties of the cells. a: Colonies of P. crispa spreading in an Antarctic habitat. b: Light spectral conditions on the surface and the middle and lower parts of a colony of $P$. crispa simulated using an HAL-100 solar

Extended Data Figure 2| Absorbance spectra of isolated proteins. Each spectrum was normalized at the peak wavelength of the red absorption band. a.u.: arbitrary unit.

Extended Data Figure 3| Amino acid sequence analysis. a: Pc-frLHC fraction after the DE53 column was loaded onto an SDS-PAGE gel and the separated proteins were stained by a CBB. The main protein band detected at $29 \mathrm{kDa}(*)$ was cut out and used for lysyl endopeptidase treatment. The fragmented peptides after lysyl endopeptidase treatment were separated by SDS-PAGE and detected by silver staining (b) or electrophoretically blotted onto a PVDF membrane (c). b: The right and left lanes were thylakoid membranes and the fragmented peptides of the $29 \mathrm{kDa}$ protein of (a), respectively. c: N-terminal 
amino acid sequences of fragmented peptides of $14,10,6$, and $4 \mathrm{kDa}$ were determined by a Procise 492cLC peptide sequencer (Applied Biosystems, Carlsbad, CA). Fragments 14 and 10 had the same N-terminal sequence.

Extended Data Figure 4| Sequence alignment of Pc-frLHC from $P$. crispa and the closely related LHCs. The deduced amino acid sequence of Pc-frLHC was classified into one of the LHCI groups along with Lhca 2 of Chlamydomonas reinhardtii (Cr_Lhca2), Lhca-J of a marine green alga, Bryopsis corticulans (Bc_LhcaJ), and Lhca 5 of a halophilic green alga, Dunaliella salina (Ds_Lhca5) with amino-acid sequence identities of $32 \%, 32 \%$, and $29 \%$, respectively. Recent analyses revealed that Cr_Lhca2, Bc_LhcaJ, and Ds_Lhca5 are orthologs and loosely bound to the side of green algal PSI in a state of a heterodimer together with Cr_Lhca9, Bc_LhcI, and Ds_Lhca6, respectively ${ }^{29,30,31,32}$. Based on these results, we concluded that Pc-frLhc is phylogenetically a member of LHCI, while it transfers excitation energy to PSII. Transmembrane helices are shaded by different colors, the signal peptides are shaded, chlorophyll-binding sites (followed by nomenclature of Liu et al.) are shown as red characters, and the sequences detected by $\mathrm{N}$-terminal sequences are shown by arrows. The signal peptides and the transmembrane helices were predicted from the 3D structures registered in the PDB (Cr_Lhca2; 6JO5, 6IJO, Bc_LhcaJ; 6IGZ, Ds_Lhca5; 6SL5) and from the results of secondary structural prediction using the Jpred 4 (doi: 10.1093/nar/gkv332) and the TargetP-2.0 (http://www.cbs.dtu.dk/services/TargetP/). The Chl708 binding site in P. crispa is in blue.

\section{Extended Data Figure 5| Phylogenetic tree of light-harvesting complexes (LHCs) of} PSI and PSII in green algae and plants. Evolutionary analyses of LHCs were conducted with the neighbor-joining method in MEGA7. We only used amino acid sequences of LHCs in which 3D structural analysis were achieved. The optimal tree with the sum of branch lengths $=17.75479796$ is shown. The percentage of replicate trees in which the associated taxa clustered together in the bootstrap test (1000 replicates) is shown next to the branches. The evolutionary distances were computed using the Poisson correction method. The rate variation among sites was modeled with a gamma distribution (shape parameter $=3$ ).

While most LHC subunits have only three transmembrane helices, a few LHC subunits, such as Cr_Lhca2, Bs_LhcaJ, and Ds_Lhca5, have been known to possess a fourth 
transmembrane helix (Four TMH).

Abbreviations: Cm: Cyanidioschyzon merolae (red alga); Cre: Chlamydomonas reinhardtii; Bc: Bryopsis corticulans; Ds: Dunaliella salina; Pc: Prasiola crispa (green algae); At: Arabidopsis thaliana (plant). Blast accession numbers: Cm_Lhcr1 (XP_005538084), Cm_Lhcr2 (XP_005537362), Cm_Lhcr3 (5ZGB_3), Cre_LHCA1 (6IJJ_1), Cre_LHCA2 (XP_001691031), Cre_LHCA3 (PNW76422), Cre_LHCA4 (6IJJ_4), Cre_LHCA5 (6IJJ_5), Cre_LHCA6 (6IJJ_6), Cre_LHCA7 (AAO16495), Cre_LHCA8 (6IJJ_8), Cre_LHCA9 (XP_001692548), Cre_CP29 (XP_001697193), Cre_PSBS2 (XP_001689923.1), Cre_CP26 (XP_001695927), Cre_LHCII (XP_001700243.1), Bc_Lhca-j (6IGZ_0), Bc_Lhca-a (6IGZ_1), Bc_Lhca-c (6IGZ_2), Bc_Lhca-d (6IGZ_3), Bc_Lhca-b (6IGZ_4), Bc_Lhca-g (6IGZ_6), Bc_Lhca-h (6IGZ_7), Bc_Lhca-i (6IGZ_9), At_Lhca1 (NP_191049.1), At_Lhca2 (NP_191706.2), At_Lhca3 (NP_001185280.1), At_Lhca4 (NP_190331.3), At_Lhca5 (NP_175137.1), At_Lhca6 (NP_173349.1), At_CP26 (NP_192772.1), At_CP29 (NP_195773.1), At_CP24 (NP_173034.1), At_LHCII (NP_174286.1), At_PSBS (NP_001319163.1), Ds_Lhca1 (6RHZ_1), Ds_Lhca2 (6RHZ_2), Ds_Lhca3 (6SL5_3), Ds_Lhca4 (6QPH_4), Ds_Lhca5 (6SL5_5), Ds_Lhca6 (6SL5_6).

Extended Data Figure 6| Cryo-EM data processing workflow. a: Representative micrograph. b: 2D averages of the particle images. c: Magnification of two top views (* and **) showing a clear indication of 11 subunits with 11-fold rotational symmetry.

Extended Data Figure 7| Summary of Cryo-EM analysis. a: Angular distribution of the cryo-EM particles. b: Gold standard Fourier shell correlation (FSC) curves of the refined $3 \mathrm{D}$ reconstruction. c: The $3 \mathrm{D}$ reconstruction is colored according to the local resolution. Top: stromal side; bottom: lumenal side. d: Representative densities of the Chl trimer. e: Map-to-model FSC curve.

Extended Data Figure 8| Identification of pigments binding to Pc-frLHC with highperformance liquid chromatography. Pigment analysis was performed with HPLC using C18-column as described in our most recent article ${ }^{37}$. The purified Pc-frLHC fraction was injected directly into the HPLC system and the absorbance at $400 \mathrm{~nm}$ was monitored. Loroxanthin (loro), violaxanthin (vio), chlorophyll $b$ (Chl $b$ ), and chlorophyll $a(\mathrm{Chl} a)$ were identified from the retention time (upper panel) and absorbance spectra 
1 (lower panel). Loroxanthin was also identified by its molecular weight (584) using liquid

2 chromatography-mass spectrometry. Three carotenoid-binding sites, L1, L2 (both

3 surrounding central helices A and B), and N1 (near helix C), are conserved among LHCs.

4 Biochemical analysis shows that Pc-frLHC binds only two carotenoids: vio and loro. The

5 vio and loro in the all-trans configuration were assigned in the L1- and L2-binding sites

6 of Pc-frLHC, respectively. The N1 site is not occupied in Pc-frLHC. a.u.: arbitrary unit. 7

8 Extended Data Table 1| Ligands of Chls in the structural model of Pc-frLHC 9

10 Extended data Table 2| Excitonic couplings $\left(\mathrm{cm}^{-1}\right)$ between $\mathrm{Chl} a$ molecules bound 11 to two subunits. Strong interactions are highlighted with hatching 
3

4

5 6 arbitrary unit. a
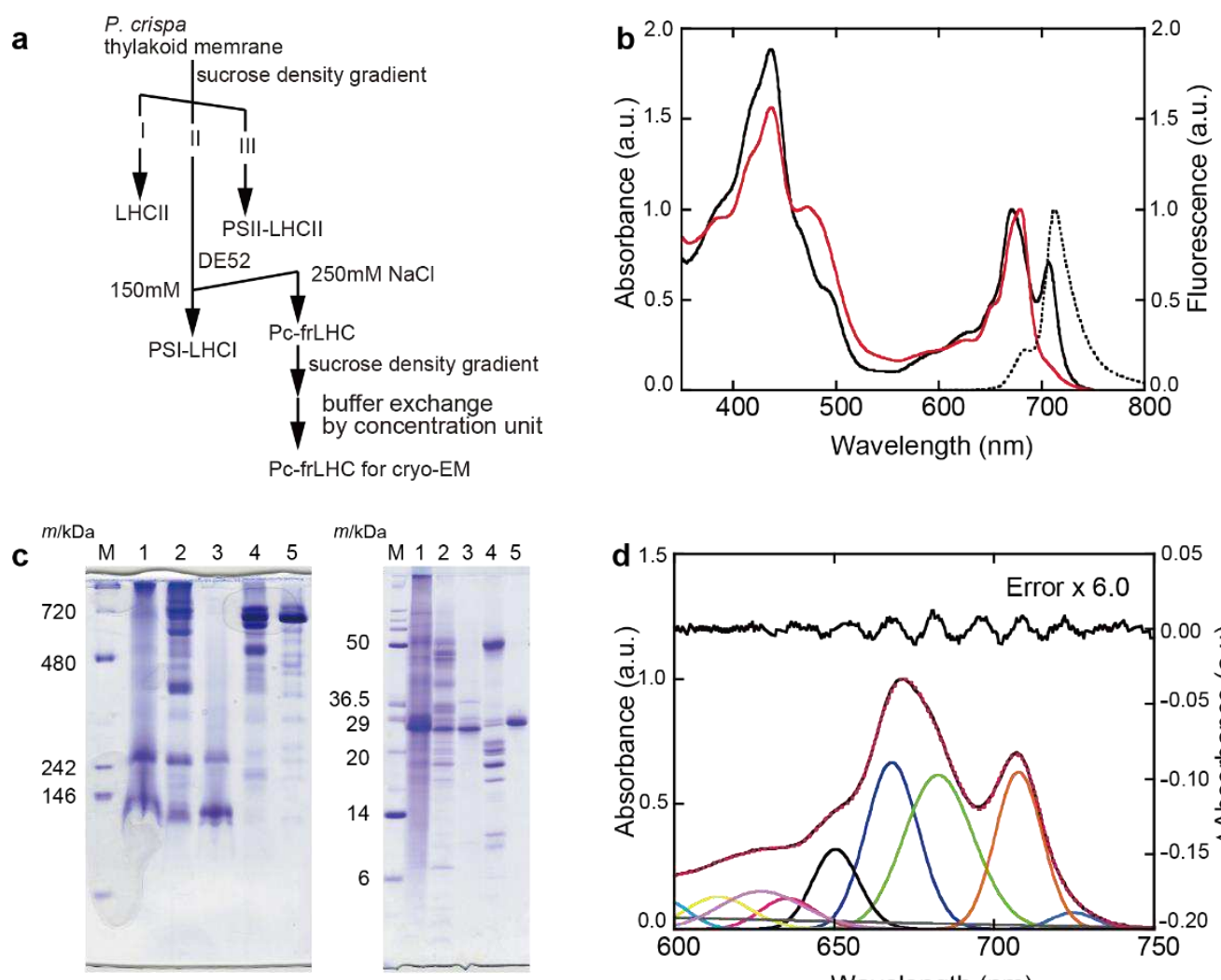

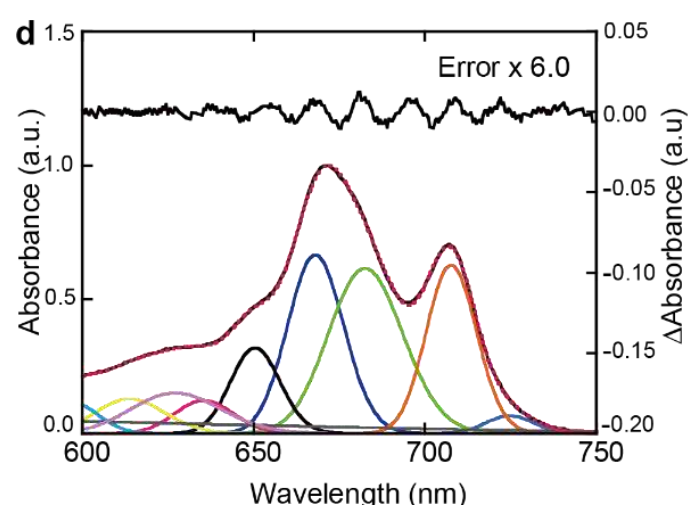

Figure 1 Purification of Pc-frLHC. a: The purification scheme of Pc-frLHC and other photosynthetic proteins from $P$. crispa's thylakoid membranes. b: Absorbance spectra (solid line) and fluorescence spectrum (dotted line) of the thylakoids (red) and Pc-frLHC (black) measured at room temperature. c: The hrCN-PAGE (left) and SDS-PAGE (right) analyses of thylakoids (lane 1), PSII-LHCII (lane 2), LHCII (lane 3), PSI-LHCI (lane 4) and Pc-frLHC (lane 5). d: The fitting analysis of the absorbance spectra of purified PcfrLHC at room temperature. The peak wavelengths of each component were estimated by the second and fourth derivative of the absorbance spectrum, and fitting analysis with Gaussian functions was performed by Magic plot 2.7.2 (Magicplot Systems). a.u.: 
a

b


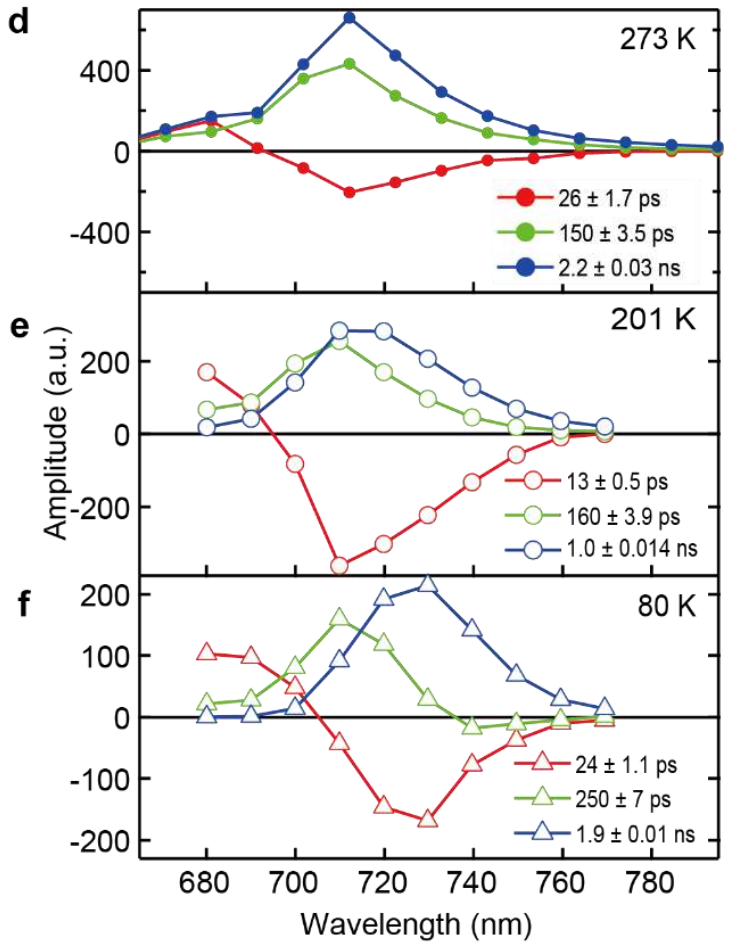

g

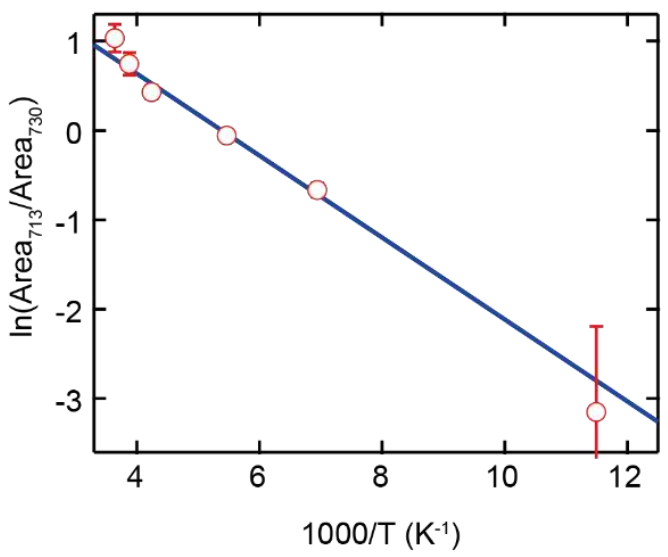

Figure 2 Spectroscopic analysis of Pc-frLHC. a,b: (a) Fluorescence time profiles of Pc-frLHC excited at $740 \mathrm{~nm}$ and monitored at $680 \mathrm{~nm}$ (green) observed at $273 \mathrm{~K}$ and (b) those excited at $460 \mathrm{~nm}$ and monitored at $680 \mathrm{~nm}$ (green), $710 \mathrm{~nm}$ (orange), and $740 \mathrm{~nm}$ (red) and observed at $80 \mathrm{~K}$. Blue curves show the fitting curves to the sum of three exponential components convolved with the instrumental response function shown by red circles in (a). c: Temperature dependence of the fluorescence spectrum of Pc-frLHC excited at $460 \mathrm{~nm}$. The blue curves are the fitting curves to the sum of two Gaussian functions (the filled green and orange curves). d-f: Fluorescence-decay-associated spectra of Pc-frLHC excited at $460 \mathrm{~nm}$ and observed at $273 \mathrm{~K}$ (d), $201 \mathrm{~K}$ (e), and $80 \mathrm{~K}$ (f). g: Analysis of the ratio Areaf713/Areaf730 using the Arrhenius equation. The blue line is the fitting according to equation S1 (see Methods) with the energy gap fixed to 318 $\mathrm{cm}^{-1}$. a.u.: arbitrary unit. 

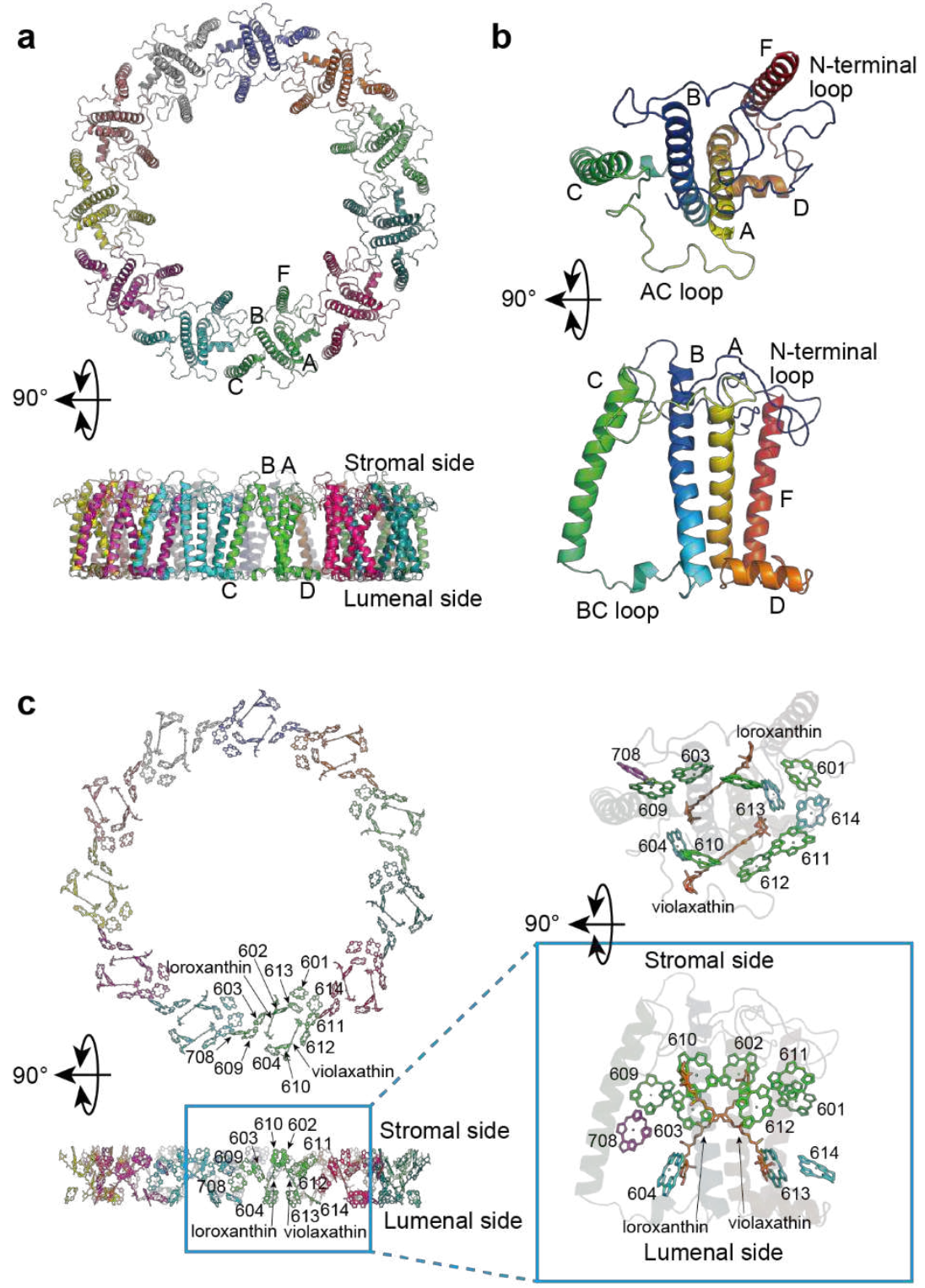

Figure 3 Overall cryo-EM structure of Pc-frLHC. a: Top (upper panel) and side views (lower panel) are shown. Each subunit is shown in a different color. b: Top and side views of the subunit of Pc-frLHC in rainbow colors from the $\mathrm{N}$-terminus in blue to $\mathrm{C}$-terminus in red. c: Chlorophyll $a$ arrangement in Pc-frLHC (the left panel). Each chlorophyll is colored in the same color as the corresponding subunit in (a). The right panel shows the arrangement of chlorophyll $a$ in the subunit. Chlorophylls on the stromal and lumenal sides are shown in green and cyan. Chl708 is shown in purple. 

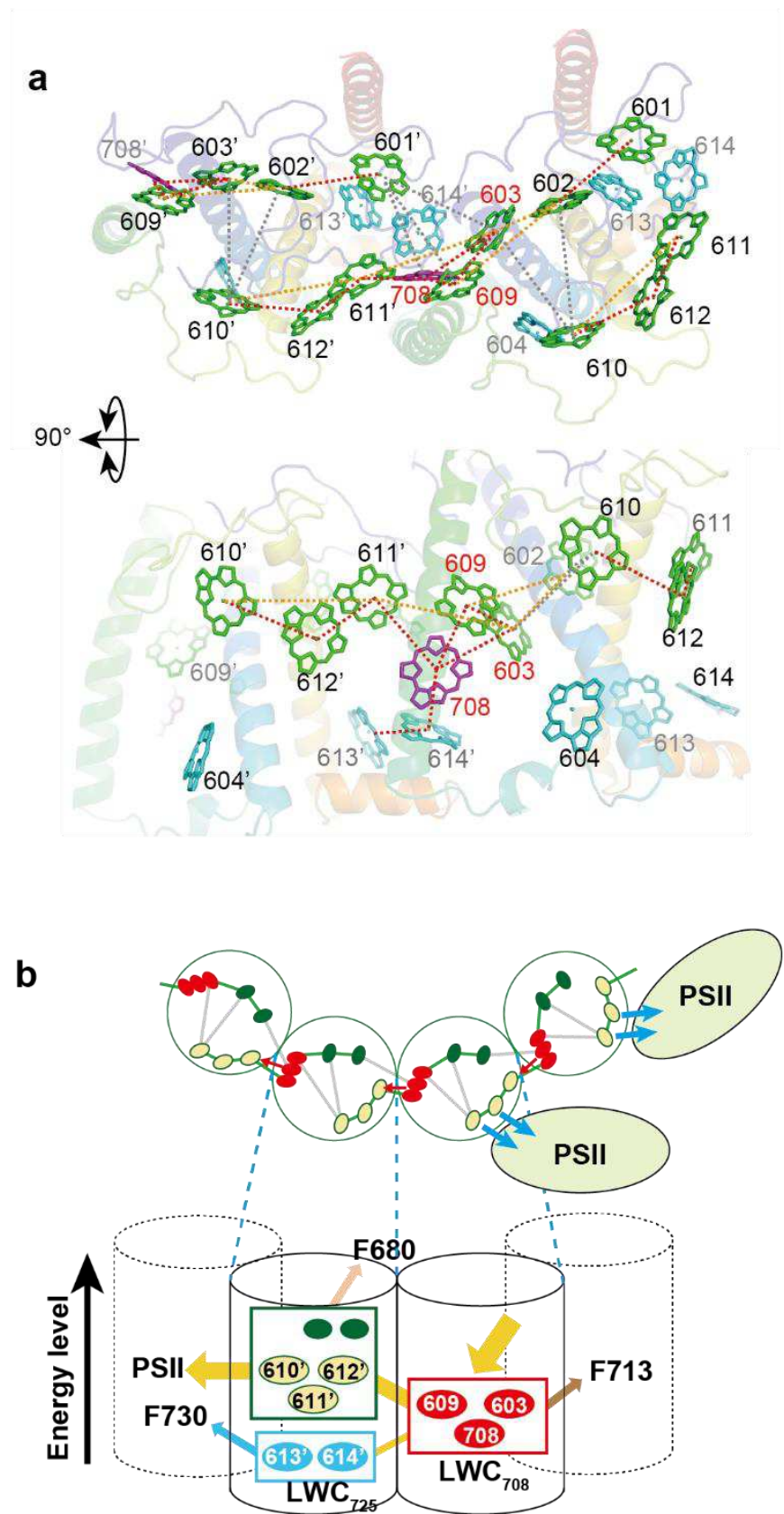

Figure 4 The chlorophyll network in Pc-frLHC. a: Connections among chlorophyll $a$ in Pc-frLHC. Energetically connected stromal chlorophylls (including Ch1708) are linked by dotted lines (Extended data Table 2). The dotted lines are colored based on the values of excitonic couplings $(E C): E C>60$ in red, $60 \geq E C>30$ in orange, and $30 \geq E C>10$ in gray. Chlorophylls on the stromal and lumenal sides are shown in green and cyan, respectively. Chl708 is shown in purple. While Chls613, 614 are energetically connected to Chls708, 612 and 601, they are partly shown in this figure for clarity. b: Schematic drawing of excited energy flows in Pc-frLHC. Pc-frLHC absorbs far red light using LWCs and distributes its energy to PSII by uphill excitation energy transfer. 


\section{Extended data}

a
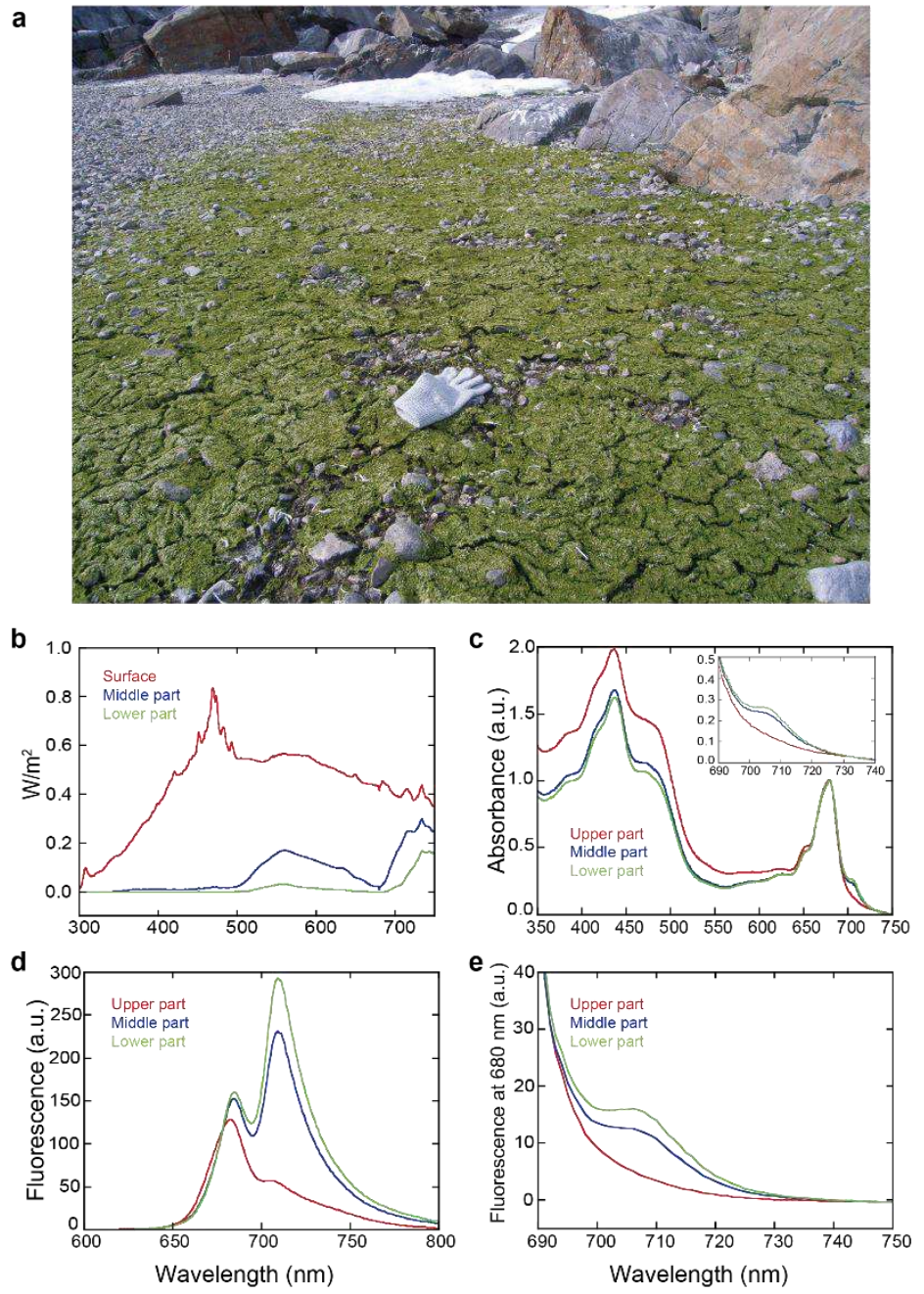

Extended Data Figure 1|Prasiola crispa in Antarctica, light conditions of the $P$. crispa colony, and spectroscopic properties of the cells. a: Colonies of $P$. crispa spreading in an Antarctic habitat. b: Light spectral conditions on the surface and the middle and lower parts of a colony of P. crispa simulated using an HAL-100 solar simulator (Asahi Spectra, Tokyo) were measured by a spectrometer, Black comet (StellarNet, Tampa, FL). A drought colony harvested from Antarctica was sliced in the upper, middle, and lower parts. Each part of a colony was sandwiched with quartz plates, respectively, and vertically set under the irradiation of the solar simulator. The light source and the transmitted light of samples were determined by a spectrometer five to ten times under each condition and averaged. c: Absorption spectra of thylakoid membranes isolated from the upper, middle, and lower parts of a colony were measured by an MPS-2450 spectrometer (Shimadzu, Kyoto, Japan). All spectra were normalized at $679 \mathrm{~nm}$. d: Emission fluorescence spectra of thylakoid membranes were measured at room temperature with excitation at $435 \mathrm{~nm}$ by an RF-6000 fluorescence spectrometer (Shimadzu). The slit widths of the excitation and emission sides were $5 \mathrm{~nm}$ and $3 \mathrm{~nm}$, respectively. e: Excitation spectra of fluorescence emission at $680 \mathrm{~nm}$ were measured by an RF-6000. Shorter wavelengths than $400 \mathrm{~nm}$ of the excitation light were cut by long-pass filters. The slit widths of the excitation and emission sides were $3 \mathrm{~nm}$ and $5 \mathrm{~nm}$, respectively. a.u.: arbitrary unit. 
Extended Data Figure 2| Absorbance spectra of isolated proteins. Each spectrum was normalized at the peak wavelength of the red absorption band. a.u.: arbitrary unit. 




\section{b}

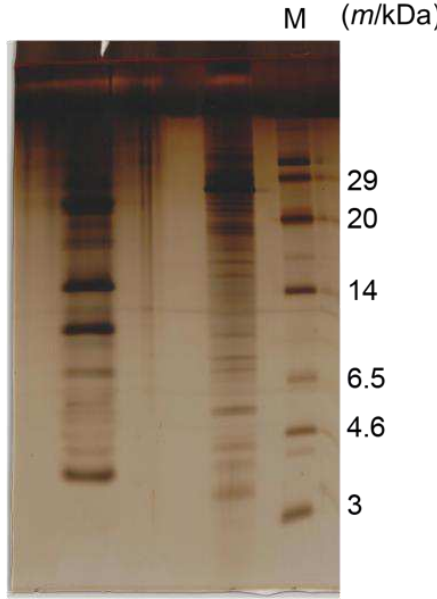

c

M $(m / k D a)$

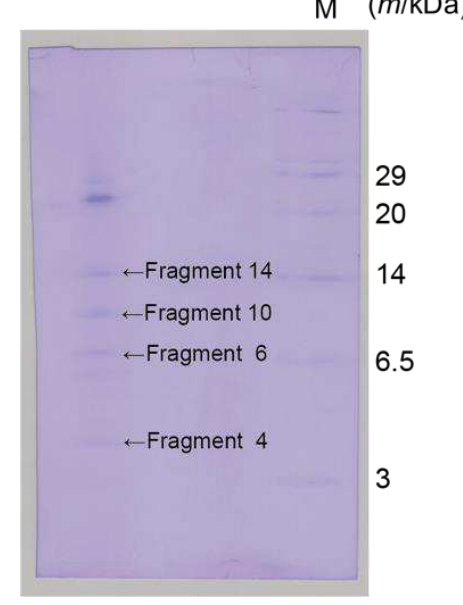

Extended Data Figure 3| Amino acid sequence analysis. a: Pc-frLHC fraction after the DE53 column was loaded onto an SDS-PAGE gel and the separated proteins were stained by a CBB. The main protein band detected at $29 \mathrm{kDa}(*)$ was cut out and used for lysyl endopeptidase treatment. The fragmented peptides after lysyl endopeptidase treatment were separated by SDSPAGE and detected by silver staining (b) or electrophoretically blotted onto a PVDF membrane (c). b: The right and left lanes were thylakoid membranes and the fragmented peptides of the 29 $\mathrm{kDa}$ protein of (a), respectively. c: $\mathrm{N}$-terminal amino acid sequences of fragmented peptides of 14, 10, 6, and $4 \mathrm{kDa}$ were determined by a Procise 492cLC peptide sequencer (Applied Biosystems, Carlsbad, CA). Fragments 14 and 10 had the same N-terminal sequence. 




Extended Data Figure 4| Sequence alignment of Pc-frLHC from $P$. crispa and the closely related LHCs. The deduced amino acid sequence of Pc-frLHC was classified into one of the LHCI groups along with Lhca 2 of Chlamydomonas reinhardtii (Cr_Lhca2), Lhca-J of a marine green alga, Bryopsis corticulans (Bc_LhcaJ), and Lhca 5 of a halophilic green alga, Dunaliella salina (Ds_Lhca5) with amino-acid sequence identities of $32 \%, 32 \%$, and $29 \%$, respectively. Recent analyses revealed that Cr_Lhca2, Bc_LhcaJ, and Ds_Lhca5 are orthologs and loosely bound to the side of green algal PSI in a state of a heterodimer together with Cr_Lhca9, Bc_LhcI, and Ds_Lhca6, respectively ${ }^{29,30,31,32}$. Based on these results, we concluded that Pc-frLhc is phylogenetically a member of LHCI, while it transfers excitation energy to PSII. Trans-membrane helices are shaded by different colors, the signal peptides are shaded, chlorophyll-binding sites (followed by nomenclature of Liu et al.) are shown as red characters, and the sequences detected by $\mathrm{N}$-terminal sequences are shown by arrows. The signal peptides and the transmembrane helices were predicted from the 3D structures registered in the PDB (Cr_Lhca2; 6JO5, 6IJO, Bc_LhcaJ; 6IGZ, Ds_Lhca5; 6SL5) and from the results of secondary structural prediction using the Jpred 4 (doi: 10.1093/nar/gkv332) and the TargetP-2.0 (http://www.cbs.dtu.dk/services/TargetP/). The Chl708 binding site in P. crispa is in blue. 
Extended Data Figure 5| Phylogenetic tree of light-harvesting complexes (LHCs) of PSI and PSII in green algae and plants. Evolutionary analyses of LHCs were conducted with the neighbor-joining method in MEGA7. We only used amino acid sequences of LHCs in which 3D structural analysis were achieved. The optimal tree with the sum of branch lengths $=17.75479796$ is shown. The percentage of replicate trees in which the associated taxa clustered together in the bootstrap test (1000 replicates) is shown next to the branches. The evolutionary distances were computed using the Poisson correction method. The rate variation among sites was modeled with a gamma distribution (shape parameter $=3$ ).

While most LHC subunits have only three transmembrane helices, a few LHC subunits, such as Cr_Lhca2, Bs_LhcaJ, and Ds_Lhca5, have been known to possess a fourth transmembrane helix (Four TMH).

Abbreviations: Cm: Cyanidioschyzon merolae (red alga); Cre: Chlamydomonas reinhardtii; Bc: Bryopsis corticulans; Ds: Dunaliella salina; Pc: Prasiola crispa (green algae); At: Arabidopsis thaliana (plant). Blast accession numbers: Cm_Lhcr1 (XP_005538084), Cm_Lhcr2 (XP_005537362), Cm_Lhcr3 (5ZGB_3), Cre_LHCA1 (6IJJ_1), Cre_LHCA2 (XP_001691031), Cre_LHCA3 (PNW76422), Cre_LHCA4 (6IJJ_4), Cre_LHCA5 (6IJJ_5), Cre_LHCA6 (6IJJ_6), Cre_LHCA7 (AAO16495), Cre_LHCA8 (6IJJ_8), Cre_LHCA9 (XP_001692548), Cre_CP29 (XP_001697193), Cre_PSBS2 (XP_001689923.1), Cre_CP26 (XP_001695927), Cre_LHCII (XP_001700243.1), Bc_Lhca-j (6IGZ_0), Bc_Lhca-a (6IGZ_1), Bc_Lhca-c (6IGZ_2), Bc_Lhcad (6IGZ_3), Bc_Lhca-b (6IGZ_4), Bc_Lhca-g (6IGZ_6), Bc_Lhca-h (6IGZ_7), Bc_Lhca-i (6IGZ_9), At_Lhca1 (NP_191049.1), At_Lhca2 (NP_191706.2), At_Lhca3 (NP_001185280.1), At_Lhca4 (NP_190331.3), At_Lhca5 (NP_175137.1), At_Lhca6 (NP_173349.1), At_CP26 (NP_192772.1), At_CP29 (NP_195773.1), At_CP24 (NP_173034.1), At_LHCII (NP_174286.1), At_PSBS (NP_001319163.1), Ds_Lhca1 (6RHZ_1), Ds_Lhca2 (6RHZ_2), Ds_Lhca3 (6SL5_3), Ds_Lhca4 (6QPH_4), Ds_Lhca5 (6SL5_5), Ds_Lhca6 (6SL5_6). 
a

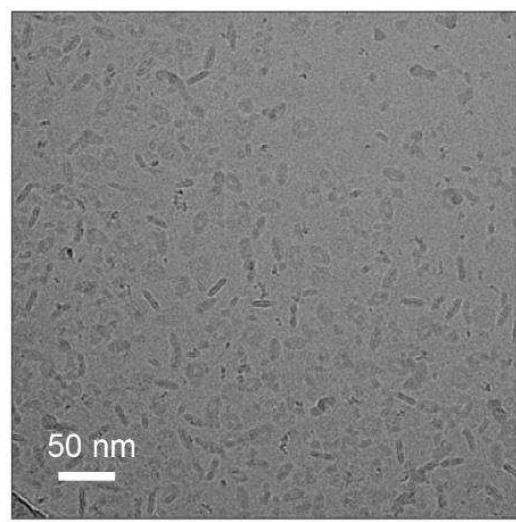

1,555 micrographs Motion correction CTF estimation Automated particle picking by crYOLO 696,095 particles<smiles>[13CH3]</smiles>
2D classification

b
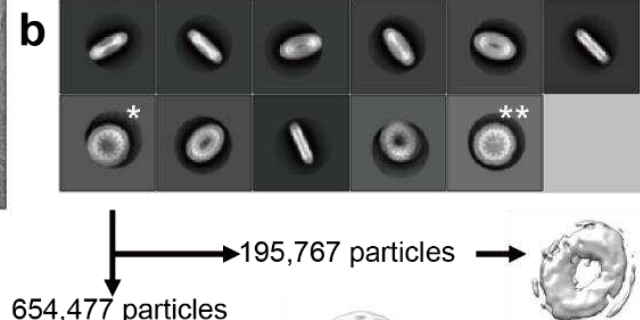

3D initial model (C1)

Resolution $3.23 \AA$

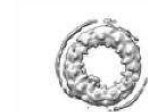

185,680 particles

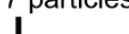

3D classification (C1)


3D classification with no alignment (C11)

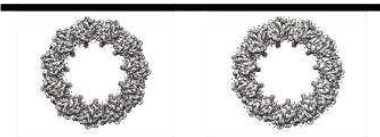

99,510 particles

$\downarrow 3 \mathrm{D}$ refinement $(\mathrm{C} 11)$

Resolution $3.13 \AA$

3

4

5

6

7

Extended Data Figure 6| Cryo-EM data processing workflow. a: Representative micrograph. b: 2D averages of the particle images. c: Magnification of two top views (* and $* *$ ) showing a clear indication of 11 subunits with 11 -fold rotational symmetry. 


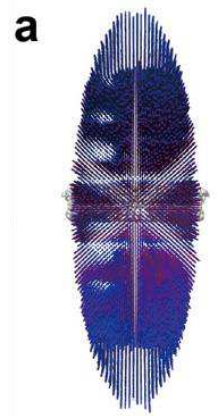

b


e
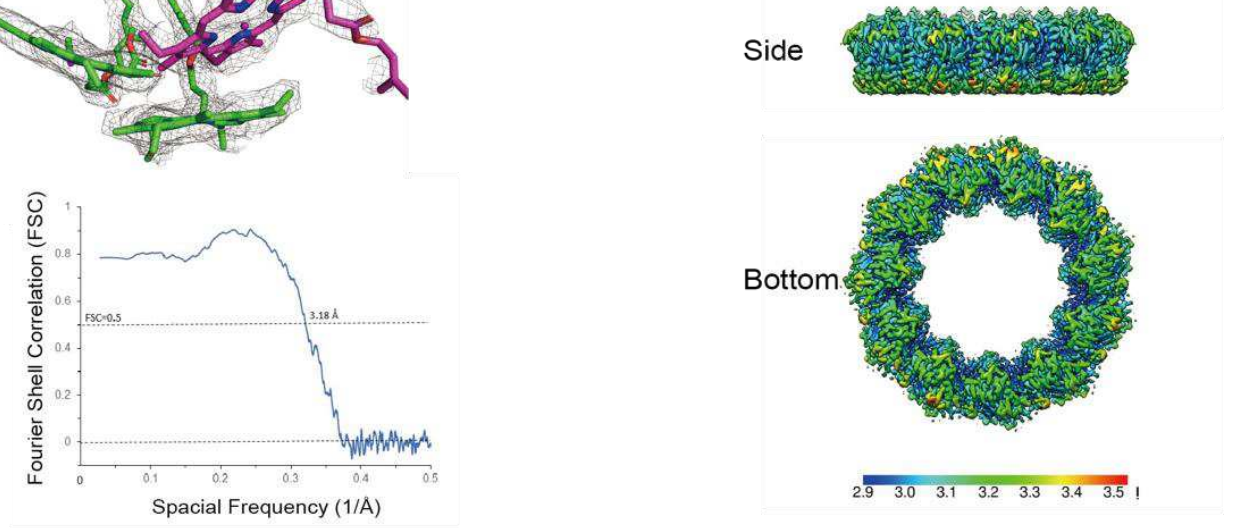

Extended Data Figure 7| Summary of Cryo-EM analysis. a: Angular distribution of the cryoEM particles. b: Gold standard Fourier shell correlation (FSC) curves of the refined 3D reconstruction. c: The 3D reconstruction is colored according to the local resolution. Top: stromal side; bottom: lumenal side. d: Representative densities of the Chl trimer. e: Map-to-model FSC curve. 



Extended Data Figure 8| Identification of pigments binding to Pc-frLHC with highperformance liquid chromatography. Pigment analysis was performed with HPLC using C18column as described in our most recent article ${ }^{37}$. The purified Pc-frLHC fraction was injected directly into the HPLC system and the absorbance at $400 \mathrm{~nm}$ was monitored. Loroxanthin (loro), violaxanthin (vio), chlorophyll $b(\mathrm{Chl} b)$, and chlorophyll $a(\mathrm{Chl} a)$ were identified from the retention time (upper panel) and absorbance spectra (lower panel). Loroxanthin was also identified by its molecular weight (584) using liquid chromatography-mass spectrometry. Three carotenoid-binding sites, L1, L2 (both surrounding central helices A and B), and N1 (near helix C), are conserved among LHCs. Biochemical analysis shows that Pc-frLHC binds only two carotenoids: vio and loro. The vio and loro in the all-trans configuration were assigned in the L1and L2-binding sites of Pc-frLHC, respectively. The N1 site is not occupied in Pc-frLHC. a.u.: arbitrary unit. 
2 Extended Data Table 1| Ligands of Chls in the structural model of Pc-frLHC

3

\begin{tabular}{|l|l|l|}
\hline Chl & Ligand & Mg-ligand distance (̊) \\
\hline 601 & unidentified & \\
\hline 602 & Glu124 & 2.77 \\
\hline 603 & Asn127 & 2.56 \\
\hline 604 & none & \\
\hline 609 & Glu178 & 2.23 \\
\hline 610 & Glu219 & 3.58 \\
\hline 611 & Glu63 & 2.62 \\
\hline 612 & Asn222 & 3.36 \\
\hline 613 & Gln236 & 2.67 \\
\hline 614 & His251 & 3.48 \\
\hline 708 & His171 & 2.59 \\
\hline
\end{tabular}

4

5 
Extended data Table 2| Excitonic couplings $\left(\mathrm{cm}^{-1}\right)$ between $\mathrm{Chl} a$ molecules bound to two subunits. Strong interactions are highlighted with hatching

\begin{tabular}{|l|ccccccccccc|}
\hline & $\mathbf{6 0 1}$ & $\mathbf{6 0 2}$ & $\mathbf{6 0 3}$ & $\mathbf{6 0 4}$ & $\mathbf{6 0 9}$ & $\mathbf{6 1 0}$ & $\mathbf{6 1 1}$ & $\mathbf{6 1 2}$ & $\mathbf{6 1 3}$ & $\mathbf{6 1 4}$ & $\mathbf{7 0 8}$ \\
\hline $\mathbf{6 0 1}$ & - & 75 & 1 & -2 & 4 & -5 & -5 & -6 & -13 & -21 & -2 \\
$\mathbf{6 0 2}$ & 75 & - & 34 & 8 & -39 & -11 & -5 & -8 & -2 & 2 & 9 \\
$\mathbf{6 0 3}$ & 1 & 34 & - & -4 & 154 & 15 & -1 & 8 & 4 & 0 & -67 \\
$\mathbf{6 0 4}$ & -2 & 8 & -4 & - & -6 & -3 & -4 & -2 & 3 & -1 & 1 \\
$\mathbf{6 0 9}$ & 4 & -39 & 154 & -6 & - & 3 & 6 & 0 & -4 & -1 & 86 \\
$\mathbf{6 1 0}$ & -5 & -11 & 15 & -3 & 3 & - & -36 & -86 & 8 & 4 & -6 \\
$\mathbf{6 1 1}$ & -5 & -5 & -1 & -4 & 6 & -36 & - & -73 & -7 & -7 & -2 \\
$\mathbf{6 1 2}$ & -6 & -8 & 8 & -2 & 0 & -86 & -73 & - & 25 & 14 & -1 \\
$\mathbf{6 1 3}$ & -13 & -2 & 4 & 3 & -4 & 8 & -7 & 25 & - & 66 & 3 \\
$\mathbf{6 1 4}$ & -21 & 2 & 0 & -1 & -1 & 4 & -7 & 14 & 66 & - & 0 \\
$\mathbf{7 0 8}$ & -2 & 9 & -67 & 1 & 86 & -6 & -2 & -1 & 3 & 0 & - \\
$\mathbf{6 0 1}$ & 2 & 1 & 15 & 1 & -11 & 3 & 0 & 1 & -2 & -1 & -15 \\
$\mathbf{6 0 2}$ & 1 & -3 & 5 & -2 & -2 & 2 & 1 & 0 & -1 & 0 & -9 \\
$\mathbf{6 0 3}$ & 0 & 1 & -1 & 1 & 1 & 0 & 0 & 0 & 0 & 0 & 0 \\
$\mathbf{6 0 4}$ & 0 & 0 & -1 & 1 & 1 & 0 & 0 & 0 & 0 & 0 & 2 \\
$\mathbf{6 0 9}$ & 0 & -1 & 2 & -1 & -2 & 1 & 0 & 0 & 0 & 0 & -1 \\
$\mathbf{6 1 0}$ & 0 & 2 & -4 & 2 & 7 & -2 & -1 & -1 & 0 & 0 & 3 \\
$\mathbf{6 1 1}$ & -2 & 9 & -33 & 6 & 65 & -6 & -2 & -1 & 1 & 1 & 64 \\
$\mathbf{6 1 2}$ & 0 & 4 & -9 & 5 & 22 & -1 & -1 & 0 & 0 & 0 & 14 \\
$\mathbf{6 1 3}$ & -1 & 2 & -9 & 3 & 9 & -2 & -1 & -1 & 2 & 1 & -7 \\
$\mathbf{6 1 4}$ & -2 & -3 & -14 & -1 & 15 & -2 & 0 & -2 & 3 & 0 & -92 \\
$\mathbf{7 0 8}$ & 0 & 0 & -1 & 0 & 0 & 0 & 0 & 0 & 0 & 0 & 1 \\
\hline
\end{tabular}




\section{Supplementary Files}

This is a list of supplementary files associated with this preprint. Click to download.

- 2pcfrlhcSIver7.2.docx 